TRANSACTIONS OF THE

AMERICAN MATHEMATICAL SOCIETY

Volume 361, Number 8, August 2009, Pages 3953-3976

S 0002-9947(09)04670-4

Article electronically published on March 12, 2009

\title{
STEADY-STATE SOLUTIONS FOR GIERER-MEINHARDT TYPE SYSTEMS WITH DIRICHLET BOUNDARY CONDITION
}

\author{
MARIUS GHERGU
}

\begin{abstract}
This paper is concerned with the following Gierer-Meinhardt type systems subject to Dirichlet boundary conditions:

$$
\begin{cases}\Delta u-\alpha u+\frac{u^{p}}{v^{q}}+\rho(x)=0, u>0, & \text { in } \Omega, \\ \Delta v-\beta v+\frac{u^{r}}{v^{s}}=0, v>0, & \text { in } \Omega, \\ u=0, v=0 & \text { on } \partial \Omega,\end{cases}
$$

where $\Omega \subset \mathbb{R}^{N}(N \geq 1)$ is a smooth bounded domain, $\rho(x) \geq 0$ in $\Omega$ and $\alpha, \beta \geq 0$. We are mainly interested in the case of different source terms, that is, $(p, q) \neq(r, s)$. Under appropriate conditions on the exponents $p, q, r$ and $s$ we establish various results of existence, regularity and boundary behavior. In the one dimensional case a uniqueness result is also presented.
\end{abstract}

\section{INTRODUCTION}

In 1972 Gierer and Meinhardt 8 proposed a mathematical model for pattern formation of spatial tissue structures in morphogenesis, a biological phenomenon discovered by Trembley [24] in 1744. The mechanism behind the Gierer-Meinhardt model is based on the existence of two chemical substances: a slowly diffusing activator and a rapidly diffusing inhibitor. The ratio of their diffusion rates is assumed to be small.

The model introduced by Gierer and Meinhardt reads as

$$
\begin{cases}u_{t}=d_{1} \Delta u-\alpha u+c \rho \frac{u^{p}}{v^{q}}+\rho_{0} \rho & \text { in } \Omega \times(0, T), \\ v_{t}=d_{2} \Delta v-\beta v+c^{\prime} \rho^{\prime} \frac{u^{r}}{v^{s}} & \text { in } \Omega \times(0, T),\end{cases}
$$

subject to Neumann boundary conditions in a smooth bounded domain $\Omega$. Here the unknowns $u$ and $v$ stand for the concentration of activator and inhibitor with the source distributions $\rho$ and $\rho^{\prime}$ respectively. In system (1.1), $d_{1}, d_{2}$ are the diffusion coefficients and $\alpha, \beta, c, c^{\prime}, \rho_{0}$ are positive constants. The exponents $p, q, r, s>0$ verify the relation $q r>(p-1)(s+1)>0$.

The model introduced by Gierer and Meinhardt has been used with satisfactory quantitative results for modelling the head regeneration process of hydra, an animal of few millimeters in length, consisting of 100,000 cells of about 15 different types and having a polar structure.

Received by the editors March 12, 2007.

2000 Mathematics Subject Classification. Primary 35J55; Secondary 35B40, 35J60.

Key words and phrases. Gierer-Meinhardt system, singular nonlinearities, asymptotic behavior. 
The Gierer-Meinhardt system originates in the Turing system 23$]$ introduced in 1952 as a mathematical model for the development of complex organisms from a single cell. It has been emphasized that localized peaks in concentration of chemical substances, known as inducers or morphogens, could be responsible for a group of cells developing differently from the surrounding cells. Turing discovered through linear analysis that a large difference in relative size of diffusivities for activating and inhibiting substances carries instability of the homogeneous, constant steady state, thus leading to the presence of nontrivial, possibly stable stationary configurations.

A global existence result for a more general system than (1.1) is given in the recent paper of Jiang [10. It has also been shown that the dynamics of the system (1.1) exhibit various interesting behaviors such as periodic solutions, unbounded oscillating global solutions, and finite time blow-up solutions. We refer the reader to Ni, Suzuki, and Takagi [18] for the entire description of dynamics concerning the system (1.1).

Many works have been devoted to the study of the steady-state solutions of (1.1); that is, solutions of the stationary system

$$
\begin{cases}d_{1} \Delta u-\alpha u+c \rho \frac{u^{p}}{v^{q}}+\rho_{0} \rho=0 & \text { in } \Omega, \\ d_{2} \Delta v-\beta v+c^{\prime} \rho^{\prime} \frac{u^{r}}{v^{s}}=0 & \text { in } \Omega,\end{cases}
$$

subject to Neumann boundary conditions. The main difficulty in the treatment of (1.2) is the lack of variational structure. Another direction of research is to consider the shadow system associated to (1.2), an idea due to Keener [11. This system is obtained by dividing by $d_{2}$ in the second equation and then letting $d_{2} \rightarrow \infty$. It has been shown that nonconstant solutions of the shadow system associated to (1.2) exhibit interior or boundary concentrating points. Among the large number of works in this direction we refer the interested reader to [19, 20, [21], 25], and [26], as well as to the survey papers of $\mathrm{Ni}[16$ ] and [17.

In this paper new features of Gierer-Meinhardt type systems are emphasized. More exactly, we shall be concerned with systems of the following type:

$$
\begin{cases}\Delta u-\alpha u+\frac{u^{p}}{v^{q}}+\rho(x)=0, u>0, & \text { in } \Omega, \\ \Delta v-\beta v+\frac{u^{r}}{v^{s}}=0, v>0, & \text { in } \Omega, \\ u=0, v=0 & \text { on } \partial \Omega,\end{cases}
$$

in a smooth bounded domain $\Omega \subset \mathbb{R}^{N}(N \geq 1)$. Here $u$ and $v$ represent the concentration of the activator and inhibitor and $\rho \in C^{0, \gamma}(\bar{\Omega})(0<\gamma<1)$ represents the source distribution of the activator. We assume that $\rho \geq 0$ in $\Omega, \rho \not \equiv 0$ and $\alpha, \beta$ are nonnegative real numbers. The case $\rho \equiv 0$ is more delicate and involves a more careful analysis of the Gierer-Meinhardt system. This situation has been analyzed in the recent works [1, 2], [18, [21, [25, and [26].

In this paper we are mainly interested in the case where the activator and inhibitor have different source terms, that is, $(p, q) \neq(r, s)$.

Let us notice that the homogeneous Dirichlet boundary condition in (1.3) (instead of Neumann's one as in (1.2) ) turns the system singular in the sense that the nonlinearities $\frac{u^{p}}{v^{q}}$ and $\frac{u^{r}}{v^{s}}$ become unbounded around the boundary.

The existent results in the literature for (1.3) concern the case of common sources of the concentrations, that is, $(p, q)=(r, s)$. If $p=q=r=s=1$ and $\rho \equiv 0$, the 
system (1.3) is studied in Choi and McKenna [1]. In Kim [12, [13] the system (1.3) is studied with $p=r$ and $q=s$. In the case of common sources, a decouplization of the system is appropriate in order to provide a priori estimates for the unknowns $u$ and $v$. More precisely, if $p=r$ and $q=s$, then subtracting the two equations in (1.3) and letting $w=u-v$ we get the following equivalent form:

$$
\begin{cases}\Delta w-\alpha w+(\beta-\alpha) w v+\rho(x)=0 & \text { in } \Omega, \\ \Delta v-\beta v+\frac{(v+w)^{p}}{v^{q}}=0 & \text { in } \Omega, \\ v=w=0 & \text { on } \partial \Omega .\end{cases}
$$

Thus, the study of system (1.3) amounts to the study of (1.4) in which the first equation is linear. This is more suitable to derive upper and lower barriers for $u$ and $v$ (see [1, 12, [13]). For more applications of the decouplization method in the context of elliptic systems we refer the reader to [14. We also mention here the paper of Choi and McKenna 2] where the existence of radially symmetric solutions in the case $p=r>1, q=1, s=0$ and $\Omega=B_{1} \subset \mathbb{R}^{2}$ is discussed. In [2], a priori bounds for concentrations $u$ and $v$ are obtained through sharp estimates for the associated Green's function.

In our case, such a decouplization is not possible due to the fact that $(p, q) \neq$ $(r, s)$. In order to overcome this lack, we shall exploit the boundary behavior of solutions of single singular equations associated to system (1.3). In turn, this approach requires uniqueness or suitable comparison principles for single singular equations that come from our system. These features are usually associated with nonlinearities having a sublinear growth, and that is why we restrict our attention to the case $p<1$. Our results extend those presented in [5], 6] and give precise answers to some questions raised in Choi and McKenna [1, [2] and Kim [12, 13]. Also the approach we give in this paper enables us to deal with various types of exponents. For instance, we shall consider the case $p<0$ (see Theorems 2.4 and 2.5) which means that the nonlinearity in the first equation of (1.3) is singular in both its variables $u$ and $v$. Furthermore, these results can be successfully applied to treat the case $-1<s \leq 0$ (see Remark 5.2).

\section{MAin Results}

We are interested in the following range of exponents:

$$
\begin{gathered}
-\infty<p<1, \\
q, r, s>0 \text { and } s \geq r-1 .
\end{gathered}
$$

In our approach we do not require any order relation between the nonnegative numbers $\alpha$ and $\beta$. Also we do not impose any growth condition on the source distribution $\rho(x)$ of the activator. A major role in our analysis will be played by the number

$$
\sigma=\min \left\{1, \frac{2+r}{1+s}\right\}
$$

First we are concerned with the case $0 \leq p<1$. The existence result in this case is the following.

Theorem 2.1. Assume that $0 \leq p<1, q \sigma<p+1$ and $q, r, s$ satisfy (2.1). Then the system (1.3) has at least one classical solution and there exist $c_{1}, c_{2}>0$ such 
that any solution $(u, v)$ of (1.3) satisfies the following estimates in $\Omega$ :

$$
c_{1} d(x) \leq u \leq c_{2} d(x) \quad \text { in } \Omega,
$$

and

$$
\begin{aligned}
c_{1} d(x) & \leq v \leq c_{2} d(x) \quad \text { if } s<r+1, \\
c_{1} d(x)(1+|\ln d(x)|)^{1 /(1+s)} & \leq v \leq c_{2} d(x)(1+|\ln d(x)|)^{1 /(1+s)} \quad \text { if } s=r+1, \\
c_{1} d(x)^{(2+r) /(1+s)} & \leq v \leq c_{2} d(x)^{(2+r) /(1+s)} \quad \text { if } s>r+1,
\end{aligned}
$$

where $d(x)=\operatorname{dist}(x, \partial \Omega)$.

Further regularity of the solution to (1.3) can be obtained using the same arguments as in Gui and Lin [9. More precisely, it is proved in 9] that if $u \in$ $C^{2}(\Omega) \cap C(\bar{\Omega})$ satisfies $-\Delta u=u^{-\nu}$ in a smooth bounded domain $\Omega$ and $u=0$ on $\partial \Omega$, then $u \in C^{1,1-\nu}(\bar{\Omega})$. Using the conclusion in Theorem 2.1 we have

Corollary 2.2. Assume that $0 \leq p<1$ and $q, r, s$ satisfy (2.1).

(i) If $q \leq p$ and $s \leq r$, then the system (1.3) has at least one classical solution. Moreover, any solution of (1.3) belongs to $C^{2}(\bar{\Omega}) \times C^{2}(\bar{\Omega})$.

(ii) If $-1<p-q<0$ and $-1<r-s<0$, then the system (1.3) has at least one classical solution. Moreover, any solution $(u, v)$ of (1.3) satisfies $u \in C^{2}(\Omega) \cap C^{1,1+p-q}(\bar{\Omega})$ and $v \in C^{2}(\Omega) \cap C^{1,1+r-s}(\bar{\Omega})$.

The issue of uniqueness is a delicate matter even in one dimension. In this case the system (1.3) reads

$$
\begin{cases}u^{\prime \prime}-\alpha u+\frac{u^{p}}{v^{q}}+\rho(x)=0 & \text { in }(0,1), \\ v^{\prime \prime}-\beta v+\frac{u^{r}}{v^{s}}=0 & \text { in }(0,1), \\ u(0)=u(1)=0, v(0)=v(1)=0 . & \end{cases}
$$

In [1] it is proved that the system (2.3) has a unique solution provided that $p=q=r=s=1$. The main idea is to write (2.3) as a linear system with smooth coefficients and then to use the $C^{2}[0,1] \times C^{2}[0,1]$ regularity of the solution. This approach has been used in [5] (see also [4] or [6. Theorem 2.7]) in the case $\beta \leq \alpha$, $0<q \leq p \leq 1$ and $r-p=s-q \geq 0$.

In this paper we are able to show that the uniqueness of the solution to (2.3) still holds provided that

$$
-1<p-q<1, \quad-1<r-s<1 .
$$

Note that for the above range of exponents, the solutions of (2.3) do not necessarily belong to $C^{2}[0,1] \times C^{2}[0,1]$. We prove that a $C^{1+\delta}$-regularity up to the boundary of the solution suffices in order to have uniqueness. Therefore, we prove

Theorem 2.3. Let $\Omega=(0,1), 0 \leq p<1$ and $q, r, s>0$ verify (2.4). Then the system (2.3) has a unique classical solution.

Unlike the Neumann boundary condition, in which a large number of multiplicities of solutions are observed, the uniqueness in the above result seems to be a particular feature of the Dirichlet boundary condition together with the sublinear character of the first equation in the system (2.3).

Next, we are concerned with the case $-\infty<p<0$. First, we prove the following nonexistence result. 
Theorem 2.4. Suppose $-\infty<p<0, q, r, s>0$ and one of the following hold:

(i) $q \geq 2$ and $s<1$;

(ii) $q>2$ and $s=1$;

(iii) $q>s+1$ and $s>1$.

Then the system (1.3) has no classical solutions.

The corresponding existence result in this case is the following.

Theorem 2.5. Assume that $-\infty<p<0, q, r, s$ satisfy (2.1) and $q \sigma<2$. Then, the system (1.3) has classical solutions. Moreover, if $q<p+1$ and $s<r+1$ any classical solution $(u, v)$ of (1.3) satisfies $u, v \in C^{2}(\Omega) \cap C^{1}(\bar{\Omega})$.

In proving Theorems 2.1 and 2.5 we rely on Schauder's fixed point theorem. The main point is to provide a priori bounds which allow us to control the map whose fixed points are the solutions of (1.3). To this aim, boundary estimates for solutions to single singular elliptic equations associated to (1.3) will be used.

The outline of the paper is as follows. In Section 3 we collect some auxiliary results concerning boundary estimates and comparison principles for elliptic equations involving singular nonlinearities. The proofs of the above results will be separately given in Sections 4 and 5 for the case $0 \leq p<1$ and $-\infty<p<0$ respectively. In the Appendix we provide an extension of Lemma 8 in [1] which is a useful tool in proving the uniqueness of the solution in one dimension.

\section{Auxiliary RESUlts}

Throughout this paper $\|\cdot\|_{\infty}$ denotes the $L^{\infty}(\Omega)$ norm. Also we denote by $\lambda_{1}$ and $\varphi_{1}$ the first eigenvalue and the first normalized eigenfunction of $-\Delta$ in $H_{0}^{1}(\Omega)$ with $\left\|\varphi_{1}\right\|_{\infty}=1$. As it is well known, $\varphi_{1} \in C^{2}(\bar{\Omega}), \varphi_{1}>0$ in $\Omega$, and there exists $C>0$ such that

$$
C d(x) \leq \varphi_{1} \leq \frac{1}{C} d(x) \quad \text { in } \Omega .
$$

We also recall the following useful result which is due to Lazer and McKenna.

Lemma 3.1 (Lazer and McKenna [15]). $\int_{\Omega} \varphi_{1}^{\tau} d x<\infty$ if and only if $\tau>-1$.

Basic to our approach is the following comparison result which is suitable for singular nonlinearities. We refer the reader to [7, Lemma 2.1] for a complete proof.

Lemma 3.2. Let $\Psi: \Omega \times(0, \infty) \rightarrow \mathbb{R}$ be a Hölder continuous function such that the mapping $(0, \infty) \ni t \longmapsto \frac{\Psi(x, t)}{t}$ is strictly decreasing for each $x \in \Omega$. Assume that there exist $v_{1}, v_{2} \in C^{2}(\Omega) \cap C(\bar{\Omega})$ such that

(a) $\Delta v_{1}+\Psi\left(x, v_{1}\right) \leq 0 \leq \Delta v_{2}+\Psi\left(x, v_{2}\right)$ in $\Omega$;

(b) $v_{1}, v_{2}>0$ in $\Omega$ and $v_{1} \geq v_{2}$ on $\partial \Omega$;

(c) $\Delta v_{1} \in L^{1}(\Omega)$ or $\Delta v_{2} \in L^{1}(\Omega)$.

Then $v_{1} \geq v_{2}$ in $\Omega$.

Another useful tool is the following result which is a direct consequence of the maximum principle.

Lemma 3.3. Let $k \in C(0, \infty)$ be a positive decreasing function and $a_{1}, a_{2} \in C(\Omega)$ with $0<a_{2} \leq a_{1}$ in $\Omega$. Assume that there exist $\beta \geq 0, v_{1}, v_{2} \in C^{2}(\Omega) \cap C(\bar{\Omega})$ such 
that $v_{1}, v_{2}>0$ in $\Omega, v_{1} \geq v_{2}$ on $\partial \Omega$ and

$$
\Delta v_{1}-\beta v_{1}+a_{1}(x) k\left(v_{1}\right) \leq 0 \leq \Delta v_{2}-\beta v_{2}+a_{2}(x) k\left(v_{2}\right) \quad \text { in } \Omega .
$$

Then $v_{1} \geq v_{2}$ in $\Omega$.

Proposition 3.4. Let $0 \leq p<1,0<q<p+1$ and $a \in C^{0, \gamma}(\Omega)(0<\gamma<1)$ be such that

$$
a_{1} \varphi_{1}^{-q}(x) \leq a(x) \leq a_{2} \varphi_{1}^{-q}(x) \quad \text { in } \Omega,
$$

for some $a_{1}, a_{2}>0$. Then, the problem

$$
\begin{cases}\Delta u-\alpha u+a(x) u^{p}+\rho(x)=0 & \text { in } \Omega, \\ u>0 & \text { in } \Omega, \\ u=0 & \text { on } \partial \Omega\end{cases}
$$

has a unique solution $u \in C^{2}(\Omega) \cap C(\bar{\Omega})$. Moreover, there exist $m_{1}, m_{2}>0$ such that

$$
m_{1} \varphi_{1} \leq u \leq m_{2} \varphi_{1} \quad \text { in } \Omega .
$$

Proof. Let $w$ be the unique solution of

$$
\begin{cases}\Delta w-\alpha w+\rho(x)=0 & \text { in } \Omega, \\ w>0 & \text { in } \Omega, \\ w=0 & \text { on } \partial \Omega .\end{cases}
$$

By standard elliptic arguments and the maximum principle we have $w \in C^{2}(\bar{\Omega})$. Obviously $\underline{u}:=w$ is a sub-solution of (3.3). Furthermore, by virtue of (3.1) we can find $c_{1}, c_{2}>0$ such that

$$
c_{1} \varphi_{1} \leq w \leq c_{2} \varphi_{1} \quad \text { in } \Omega .
$$

Since $q<p+1$, by a result in Wei [27, there exists $h \in C^{2}(0,1) \cap C^{1}[0,1]$ such that

$$
\begin{cases}-h^{\prime \prime}(t)=t^{-q} h^{p}(t), & \text { for all } 0<t<1, \\ h>0 & \text { in }(0,1), \\ h(0)=h(1)=0 . & \end{cases}
$$

Using the fact that $h^{\prime}(0)>0$ we have

$$
c_{3} t \leq h(t) \leq c_{4} t
$$

for $t>0$ small enough and for some $c_{3}, c_{4}>0$. Furthermore, we may find $c>0$ such that $h^{\prime}\left(c \varphi_{1}\right)>0$ in $\bar{\Omega}$.

We are looking for a super-solution of (3.3) in the form $\bar{u}:=M h\left(c \varphi_{1}\right)+w$, for $M>1$ large enough. For this purpose we have to check that the inequality $-\Delta \bar{u}+\alpha \bar{u} \geq a(x) \bar{u}^{p}+\rho(x)$ holds in $\Omega$ provided that $M>1$ is sufficiently large.

We have

$$
\begin{aligned}
-\Delta \bar{u}+\alpha \bar{u} & \geq-\Delta\left(M h\left(c \varphi_{1}\right)\right)+\rho(x) \\
& =M c^{2-q} \varphi_{1}^{-q} h^{p}\left(c \varphi_{1}\right)\left|\nabla \varphi_{1}\right|^{2}+M \lambda_{1} c \varphi_{1} h^{\prime}\left(c \varphi_{1}\right)+\rho(x) \quad \text { in } \Omega .
\end{aligned}
$$

By (3.7) we may write

$$
-\Delta \bar{u}+\alpha \bar{u} \geq M c^{2+p-q} c_{3}^{p} \varphi_{1}^{p-q}\left|\nabla \varphi_{1}\right|^{2}+M \lambda_{1} c \varphi_{1} h^{\prime}\left(c \varphi_{1}\right)+\rho(x) \quad \text { in } \Omega .
$$

On the other hand, by (3.2), (3.6) and (3.7) we have

$$
a(x) \bar{u}^{p} \leq a_{2} \varphi_{1}^{-q}\left(M h\left(c \varphi_{1}\right)+w\right)^{p} \leq a_{2} \varphi_{1}^{p-q}\left(M c c_{4}+c_{2}\right)^{p} \quad \text { in } \Omega .
$$


Using Hopf's maximum principle, there exist $\omega \subset \subset \Omega$ and $\delta>0$ such that

$$
\left|\nabla \varphi_{1}\right|>\delta \text { in } \Omega \backslash \omega \text { and } \varphi_{1}>\delta \text { in } \omega .
$$

Since $0 \leq p<1$, we may choose $M>1$ such that

$$
\begin{gathered}
M c^{2+p-q} c_{3}^{p} \delta^{2}>a_{2}\left(M c c_{4}+c_{2}\right)^{p}, \\
M \lambda_{1} c \min _{\bar{\omega}} \varphi_{1} h^{\prime}\left(c \varphi_{1}\right) \geq a_{2}\left(M c c_{4}+c_{2}\right)^{p} \max _{\bar{\omega}} \varphi_{1}^{p-q} .
\end{gathered}
$$

Combining (3.9), (3.10) and (3.12) we obtain

$$
-\Delta \bar{u}+\alpha \bar{u} \geq M c^{2+p-q} c_{3}^{p} \varphi_{1}^{p-q}\left|\nabla \varphi_{1}\right|^{2}+\rho(x) \geq a(x) \bar{u}^{p}+\rho(x) \quad \text { in } \Omega \backslash \omega .
$$

Furthermore, by (3.9), (3.10) and (3.13) we deduce

$$
-\Delta \bar{u}+\alpha \bar{u} \geq M \lambda_{1} c \varphi_{1} h^{\prime}\left(c \varphi_{1}\right)+\rho(x) \geq a(x) \bar{u}^{p}+\rho(x) \quad \text { in } \omega .
$$

Now the claim follows by (3.14) and (3.15). Thus, the problem (3.3) has a solution $u \in C^{2}(\Omega) \cap C(\bar{\Omega})$ such that $\underline{u} \leq u \leq \bar{u}$ in $\Omega$. By (3.6) and (3.7) we obtain the estimate (3.4). This also implies that

$$
C_{1} \varphi_{1}^{p-q} \leq a(x) u^{p} \leq C_{2} \varphi_{1}^{p-q} \quad \text { in } \Omega
$$

for some $C_{1}, C_{2}>0$. Since $p-q>-1$, by Lemma 3.1 we get $a(x) u^{p} \in L^{1}(\Omega)$ which finally yields $\Delta u \in L^{1}(\Omega)$. Now the uniqueness follows by Lemma 3.2. This concludes the proof of Proposition 3.4.

We next consider the problem

$$
\begin{cases}\Delta v-\beta v+a(x) v^{-s}+b(x)=0 & \text { in } \Omega, \\ v>0 & \text { in } \Omega, \\ v=0 & \text { on } \partial \Omega,\end{cases}
$$

where $a \in C^{0, \gamma}(\Omega)(0<\gamma<1)$ satisfies

$$
a_{1} \varphi_{1}^{r}(x) \leq a(x) \leq a_{2} \varphi_{1}^{r}(x) \quad \text { in } \Omega,
$$

for some $a_{1}, a_{2}>0$ and $r \in \mathbb{R}$. We also assume that $b \in C^{0, \gamma}(\bar{\Omega}), \beta \geq 0$ and $s>0$.

For convenience, let us introduce $\Gamma_{s, r}:(0, \infty) \rightarrow(0, \infty)$ defined by

$$
\Gamma_{s, r}(t)= \begin{cases}t, & \text { if } s<r+1, \\ t(1+|\log t|)^{1 /(1+s)}, & \text { if } s=r+1, \\ t^{(2+r) /(1+s)}, & \text { if } s>r+1,\end{cases}
$$

for all $r>-2$ and $s>0$. It is easy to see that

$$
\Gamma_{s, r}(t) \geq t^{\sigma} \quad \text { for all } t>0,
$$

where $\sigma$ is defined in (2.2). Moreover, for all $m>0$ there exists $m_{1}, m_{2}>0$ such that

$$
m_{1} \Gamma_{s, r}(t) \leq \Gamma_{s, r}(m t) \leq m_{2} \Gamma_{s, r}(t) \quad \text { for all } t>0 .
$$

Proposition 3.5. (i) If $r \leq-2$, then the problem (3.16) has no classical solutions.

(ii) If $r>-2$, then the problem (3.16) has a unique solution $u \in C^{2}(\Omega) \cap C(\bar{\Omega})$. Moreover, there exist $c_{1}, c_{2}>0$ such that

$$
c_{1} \Gamma_{r, s}\left(\varphi_{1}\right) \leq v \leq c_{2} \Gamma_{r, s}\left(\varphi_{1}\right) \quad \text { in } \Omega .
$$


A general nonexistence result for singular elliptic equations with unbounded potentials can be found in [3. Also a nonexistence result in the case $b \equiv 0, \beta=0$ and $r \leq-2$ is presented in [28, Theorem 1.2]. Concerning the existence part in Proposition 3.5, a similar result can be found in [9] in the case $b \equiv 0, \beta=0$ and $r \geq 0$. Here we shall give a different proof which relies on a direct construction of a sub- and super-solution. This will provide the estimate (3.21).

Proof. (i) Assume that there exist $r \geq-2$ and $v \in C^{2}(\Omega) \cap C(\bar{\Omega})$ a classical solution of (3.16). For $0<\varepsilon<1$ consider the problem

$$
\begin{cases}\Delta z-\beta z+a_{1}\left(\varphi_{1}+\varepsilon\right)^{r}(z+\varepsilon)^{-s}=0 & \text { in } \Omega, \\ z>0 & \text { in } \Omega, \\ z=0 & \text { on } \partial \Omega .\end{cases}
$$

Obviously, $\underline{z}=0$ is a sub-solution and $\bar{z}=v$ is a super-solution of (3.22). Hence, for all $0<\varepsilon<1$ there exists $z_{\varepsilon} \in C^{2}(\bar{\Omega})$ a solution of (3.22) such that $0<z_{\varepsilon} \leq v$ in $\Omega$. Multiplying by $\varphi_{1}$ in (3.22) and then integrating over $\Omega$ we get

$$
\left(\beta+\lambda_{1}\right) \int_{\Omega} z_{\varepsilon} \varphi_{1} d x=a_{1} \int_{\Omega} \varphi_{1}\left(\varphi_{1}+\varepsilon\right)^{r}\left(z_{\varepsilon}+\varepsilon\right)^{-s} d x .
$$

Since $z_{\varepsilon} \leq v$ in $\Omega$, the above equality yields

$$
\left(\beta+\lambda_{1}\right) \int_{\Omega} v \varphi_{1} d x \geq a_{1}\left(1+\|v\|_{\infty}\right)^{-s} \int_{\Omega} \varphi_{1}\left(\varphi_{1}+\varepsilon\right)^{r} d x
$$

This implies

$$
\int_{\omega} \varphi_{1}\left(\varphi_{1}+\varepsilon\right)^{r} d x<M \quad \text { for all } \omega \subset \subset \Omega
$$

where $M>0$ does not depend on $\varepsilon$. Passing to the limit with $\varepsilon \rightarrow 0$ in the above inequality we find $\int_{\omega} \varphi_{1}^{1+r} d x<M$, for all $\omega \subset \subset \Omega$; that is, $\int_{\Omega} \varphi_{1}^{1+r} d x<\infty$. Since $r \geq-2$, the last inequality contradicts Lemma 3.1. Therefore, the problem (3.16) has no classical solutions if $r \geq-2$.

(ii) Let $r>-2$ and $s \geq r-1$. According to [22, Theorem 1], there exists $H \in C^{2}(0,1) \cap C[0,1]$ such that

$$
\begin{cases}-H^{\prime \prime}(t)=t^{r} H^{-s}(t), & \text { for all } 0<t<1, \\ H>0 & \text { in }(0,1), \\ H(0)=H(1)=0 . & \end{cases}
$$

Since $H$ is concave, there exists $H^{\prime}(0+)>0$. Hence, taking $0<\eta<1$ sufficiently small, we can assume that $H^{\prime}>0$ in $(0, \eta)$. From [22, p. 904] (see also Theorem 3.5 in [3]), there exist $c_{1}, c_{2}>0$ such that

$$
c_{1} \Gamma_{s, r}(t) \leq H(t) \leq c_{2} \Gamma_{s, r}(t) \quad \text { in }(0, \eta) .
$$

As a consequence of (3.24) and the fact that $s \geq r-1$ we derive

$$
H(t)^{s+1} \leq c_{3} t^{r} \quad \text { in }(0, \eta)
$$

for some positive constant $c_{3}>0$. Let $c>0$ be such that $c \varphi_{1}<\eta$ in $\Omega$. We claim that we can find $0<m<1$ small enough such that $\underline{v}:=m H\left(c \varphi_{1}\right)$ satisfies

$$
2 \beta \underline{v}^{1+s} \leq a(x) \quad \text { in } \Omega \quad \text { and } \quad-\Delta \underline{v} \leq \frac{1}{2} a(x) \underline{v}^{-s} \quad \text { in } \Omega .
$$


Then, from (3.26) we deduce

$$
\Delta \underline{v}-\beta \underline{v}+a(x) \underline{v}^{-s} \geq 0 \quad \text { in } \Omega
$$

that is, $\underline{v}$ is a sub-solution of (3.16).

By virtue of (3.17) and (3.25) we have

$$
2 \beta \underline{v}^{1+s}=2 \beta m^{1+s} H^{1+s}\left(c \varphi_{1}\right) \leq 2 \beta m^{1+s} c_{3}\left(c \varphi_{1}\right)^{r} \leq \frac{2 \beta m^{1+s} c^{r} c_{3}}{a_{1}} a(x) \quad \text { in } \Omega .
$$

Let us now choose $m>0$ such that $2 \beta m^{1+s} c^{r} c_{3}<a_{1}$. This concludes the first inequality in (3.26).

In order to establish the second inequality in (3.26), a straightforward computation yields

$$
\begin{aligned}
-\Delta \underline{v} & =-m c^{2}\left|\nabla \varphi_{1}\right|^{2} H^{\prime \prime}\left(c \varphi_{1}\right)+m \lambda_{1} c \varphi_{1} H^{\prime}\left(c \varphi_{1}\right) \\
& =m c^{2+r} \varphi_{1}^{r}\left|\nabla \varphi_{1}\right|^{2} H^{-s}\left(c \varphi_{1}\right)+m \lambda_{1} c \varphi_{1} H^{\prime}\left(c \varphi_{1}\right) \\
& =m^{1+s} c^{2+r} \varphi_{1}^{r}\left|\nabla \varphi_{1}\right|^{2} \underline{v}^{-s}+m \lambda_{1} c \varphi_{1} H^{\prime}\left(c \varphi_{1}\right) \quad \text { in } \Omega .
\end{aligned}
$$

Since $H^{\prime}$ is decreasing on $(0, \eta)$, it follows that $t H^{\prime}(t) \leq H(t)$ for all $t \in(0, \eta)$. Furthermore, from (3.25) we deduce

$$
c \varphi_{1} H^{\prime}\left(c \varphi_{1}\right) \leq H\left(c \varphi_{1}\right) \leq c_{3}\left(c \varphi_{1}\right)^{r} H^{-s}\left(c \varphi_{1}\right) \quad \text { in } \Omega .
$$

Combining (3.28) and (3.29), for $0<m<1$ we obtain

$$
\begin{aligned}
-\Delta \underline{v} & \leq m^{1+s} c^{2+r} \varphi_{1}^{r}\left|\nabla \varphi_{1}\right|^{2} \underline{v}^{-s}+m \lambda_{1} c^{r} c_{3} \varphi_{1}^{r} H^{-s}\left(c \varphi_{1}\right) \\
& \leq m c^{2+r} \varphi_{1}^{r}\left|\nabla \varphi_{1}\right|^{2} \underline{v}^{-s}+m \lambda_{1} c^{r} c_{3} \varphi_{1}^{r} \underline{v}^{-s} \\
& =m c^{r} \varphi_{1}^{r} \underline{v}^{-s}\left(c^{2}\left|\nabla \varphi_{1}\right|^{2}+c_{3} \lambda_{1}\right) \\
& \leq \frac{m c^{r}}{a_{1}}\left(c^{2}\left\|\nabla \varphi_{1}\right\|_{\infty}^{2}+c_{3} \lambda_{1}\right) a(x) \underline{v}^{-s} \quad \text { in } \Omega .
\end{aligned}
$$

Now, it suffices to choose $0<m<1$ such that $\frac{m c^{r}}{a_{1}}\left(c^{2}\left\|\nabla \varphi_{1}\right\|_{\infty}^{2}+c_{3} \lambda_{1}\right)<\frac{1}{2}$. This establishes the second inequality in (3.26) and the fact that $\underline{v}$ is a sub-solution of (3.16).

Next we provide a super-solution $\bar{v}$ of (3.16) such that $\underline{v} \leq \bar{v}$ in $\Omega$. To this aim we first claim that there exists $M>1$ large enough such that $z:=M H\left(c \varphi_{1}\right)$ satisfies

$$
\Delta z+a(x) z^{-s} \leq 0 \quad \text { in } \Omega .
$$

As before we have

$$
\Delta z=-M^{1+s} c^{2+r} \varphi_{1}^{r}\left|\nabla \varphi_{1}\right|^{2} z^{-s}-M \lambda_{1} c \varphi_{1} H^{\prime}\left(c \varphi_{1}\right) \quad \text { in } \Omega .
$$

Let $\omega \subset \subset \Omega$ and $\delta>0$ be such that (3.11) holds, and let us consider $M>1$ such that

$$
\begin{gathered}
M^{1+s} c^{2+r} \delta^{2}>a_{2}, \\
M^{1+s} c \lambda_{1} \min _{\bar{\omega}} \varphi_{1} H^{\prime}\left(c \varphi_{1}\right) \geq a_{2} \max _{\bar{\omega}} \varphi_{1}^{r} H^{-s}\left(c \varphi_{1}\right) .
\end{gathered}
$$


Then, as in the proof of Proposition 3.4, by (3.11) and (3.32)-(3.33) we get

$$
\begin{aligned}
\Delta z+a(x) z^{-s} & \leq-M^{1+s} c^{2+r} \varphi_{1}^{r}\left|\nabla \varphi_{1}\right|^{2} z^{-s}+a(x) z^{-s} \\
& \leq-M^{1+s} c^{2+r} \delta^{2} \varphi_{1}^{r} z^{-s}+a_{2} \varphi_{1}^{r} z^{-s} \\
& =-\left(M^{1+s} c^{2+r} \delta^{2}-a_{2}\right) \varphi_{1}^{r} z^{-s} \leq 0 \quad \text { in } \Omega \backslash \omega
\end{aligned}
$$

and

$$
\begin{aligned}
\Delta z+a(x) z^{-s} & \leq-M \lambda_{1} c \varphi_{1} H^{\prime}\left(c \varphi_{1}\right)+a(x) z^{-s} \\
& \leq-M \lambda_{1} c \varphi_{1} H^{\prime}\left(c \varphi_{1}\right)+a_{2} \varphi_{1}^{r} z^{-s} \\
& =-\frac{1}{M^{s}}\left(M^{1+s} c \lambda_{1} \varphi_{1} H^{\prime}\left(c \varphi_{1}\right)-a_{2} \varphi_{1}^{r} H^{-s}\left(c \varphi_{1}\right)\right) \\
& \leq 0 \quad \text { in } \omega .
\end{aligned}
$$

Hence, we have obtained the inequality in (3.30).

Let $\tilde{w} \in C^{2}(\bar{\Omega})$ be the unique solution of

$$
\begin{cases}\Delta \tilde{w}-\beta \tilde{w}+b(x)=0 & \text { in } \Omega, \\ \tilde{w}>0 & \text { in } \Omega, \\ \tilde{w}=0 & \text { on } \partial \Omega .\end{cases}
$$

Then $\bar{v}:=z+\tilde{w}$ satisfies $\bar{v}>0$ in $\Omega$ and $\bar{v}=0$ on $\partial \Omega$, and by (3.30) we have

$$
\Delta \bar{v}-\beta \bar{v}+a(x) \bar{v}^{-s}+b(x) \leq \Delta z-\beta z+a(x) z^{-s} \leq 0 \quad \text { in } \Omega .
$$

Hence, $\bar{v}$ is a super-solution of (3.16), and clearly we have $\underline{v} \leq \bar{v}$ in $\Omega$. It follows that problem (3.16) has a classical solution $v \in C^{2}(\Omega) \cap C(\bar{\Omega})$ such that $\underline{v} \leq v \leq \bar{v}$ in $\Omega$.

On the other hand, since $\tilde{w} \in C^{2}(\bar{\Omega})$, we deduce that there exists $\tilde{c}_{1}>0$ such that $\tilde{w} \leq \tilde{c}_{1} \varphi_{1}$ in $\Omega$. This implies $\tilde{w} \leq \tilde{c}_{2} \Gamma_{s, r}\left(\varphi_{1}\right)$ in $\Omega$, for some $\tilde{c}_{2}>0$. Finally, using the last inequality, the definition of $\underline{v}, \bar{v}$ and (3.24), we get the estimate (3.21). The uniqueness of the solution follows by Lemma 3.3. This completes the proof of Proposition 3.5

\section{The CASE $0 \leq p<1$}

4.1. Proof of Theorem 2.1, Let $0<\varepsilon_{0}<1$ and set

$$
\Omega_{\varepsilon}:=\{x \in \Omega: d(x)>\varepsilon\}, \quad \text { for all } 0<\varepsilon<\varepsilon_{0} .
$$

For $\varepsilon_{0}$ small enough, $\Omega_{\varepsilon}$ remains a smooth domain. The existence of a solution to (1.3) will be proved by considering the approximated system

$$
\begin{cases}\Delta u-\alpha u+\frac{u^{p}}{v^{q}}+\rho(x)=0, u>0, & \text { in } \Omega_{\varepsilon}, \\ \Delta v-\beta v+\frac{u^{r}}{v^{s}}=0, v>0, & \text { in } \Omega_{\varepsilon}, \\ u=\varepsilon, v=\Gamma_{s, r}(\varepsilon) & \text { on } \partial \Omega_{\varepsilon} .\end{cases}
$$

The existence of a classical solution to (4.2) is obtained by using Schauder's fixed point theorem. For $0<\varepsilon<\varepsilon_{0}$ and $m_{1}, m_{2}<1<M_{1}, M_{2}$ consider

$$
\mathcal{A}_{\varepsilon}=\left\{\begin{array}{r}
m_{1} \varphi_{1} \leq u \leq M_{1} \varphi_{1} \text { in } \Omega_{\varepsilon}, \\
(u, v) \in C\left(\bar{\Omega}_{\varepsilon}\right) \times C\left(\bar{\Omega}_{\varepsilon}\right): m_{2} \Gamma_{s, r}\left(\varphi_{1}\right) \leq v \leq M_{2} \Gamma_{s, r}\left(\varphi_{1}\right) \text { in } \Omega_{\varepsilon}, \\
u=\varepsilon, v=\Gamma_{s, r}(\varepsilon) \text { on } \partial \Omega_{\varepsilon}
\end{array}\right\} .
$$


Next, we define the mapping $\mathcal{T}: \mathcal{A}_{\varepsilon} \rightarrow C\left(\bar{\Omega}_{\varepsilon}\right) \times C\left(\bar{\Omega}_{\varepsilon}\right)$ as follows. For $(u, v) \in \mathcal{A}_{\varepsilon}$ we set

$$
\mathcal{T}(u, v)=(T u, T v)
$$

where $T u$ and $T v$ satisfy

$$
\begin{cases}\Delta(T u)-\alpha(T u)+\frac{(T u)^{p}}{v_{r}^{q}}+\rho(x)=0, T u>0, & \text { in } \Omega_{\varepsilon}, \\ \Delta(T v)-\beta(T v)+\frac{u^{r}}{(T v)^{s}}=0, T v>0, & \text { in } \Omega_{\varepsilon}, \\ T u=\varepsilon, T v=\Gamma_{s, r}(\varepsilon) & \text { on } \partial \Omega_{\varepsilon} .\end{cases}
$$

Using the definition of $\mathcal{A}_{\varepsilon}$, by sub- and super-solution methods combined with Lemma 3.2 and Lemma 3.3 , the above system has a unique solution $(T u, T v)$ with $T u, T v \in C^{2}\left(\bar{\Omega}_{\varepsilon}\right)$. Basic to our approach are the following two results which allow us to apply Schauder's fixed point theorem.

Lemma 4.1. There exist $m_{1}<1<M_{1}$ and $m_{2}<1<M_{2}$ which are independent of $\varepsilon$ such that $\mathcal{T}\left(\mathcal{A}_{\varepsilon}\right) \subseteq \mathcal{A}_{\varepsilon}$, for all $0<\varepsilon<\varepsilon_{0}$.

Proof. Let $w \in C^{2}(\bar{\Omega})$ be the unique solution of problem (3.5). In view of (3.1) and (3.6) we have

$$
w(x) \leq c_{2} \varphi_{1} \leq \frac{c_{2}}{C} d(x)=\frac{c_{2}}{C} \varepsilon \quad \text { on } \partial \Omega_{\varepsilon} .
$$

Hence, if $\delta_{1}=\min \left\{1, \frac{C}{c_{2}}\right\}$, then $\delta_{1} w \leq \varepsilon$ on $\partial \Omega_{\varepsilon}$. Furthermore,

$$
\begin{gathered}
\Delta(T u)-\alpha(T u)+\rho(x) \leq 0 \leq \Delta\left(\delta_{1} w\right)-\alpha\left(\delta_{1} w\right)+\rho(x) \quad \text { in } \Omega_{\varepsilon}, \\
T u=\varepsilon \geq \delta_{1} w \quad \text { on } \partial \Omega_{\varepsilon} .
\end{gathered}
$$

By maximum principle, we obtain $T u \geq \delta_{1} w$ in $\Omega_{\varepsilon}$. In view of (3.6), let us choose $m_{1}=\delta_{1} c_{1}$ in the definition of $\mathcal{A}_{\varepsilon}$ (where $c_{1}$ is the constant in (3.6)). Then, (3.6) combined with the last estimates yields

$$
T u \geq m_{1} \varphi_{1} \quad \text { in } \Omega_{\varepsilon} .
$$

From the second equation in (4.4) and the fact that $u \geq m_{1} \varphi_{1}$ in $\Omega_{\varepsilon}$, we have

$$
\Delta(T v)-\beta(T v)+\frac{m_{1}^{r} \varphi_{1}^{r}}{(T v)^{s}} \leq 0 \quad \text { in } \Omega_{\varepsilon} .
$$

Let us consider the problem

$$
\begin{cases}\Delta \xi-\beta \xi+\varphi_{1}^{r} \xi^{-s}=0 & \text { in } \Omega, \\ \xi>0 & \text { in } \Omega, \\ \xi=0 & \text { on } \partial \Omega .\end{cases}
$$

Using Proposition 3.5 (ii), there exists $\xi \in C^{2}(\Omega) \cap C(\bar{\Omega})$, a unique solution of (4.7), with the additional property

$$
c_{3} \Gamma_{s, r}\left(\varphi_{1}\right) \leq \xi \leq c_{4} \Gamma_{s, r}\left(\varphi_{1}\right) \text { in } \Omega,
$$

for some $c_{3}, c_{4}>0$. Moreover, by (4.8), (3.1) and the property (3.20) of $\Gamma_{s, r}$ we can find $c_{5}, c_{6}>0$ such that

$$
c_{5} \Gamma_{s, r}(d(x)) \leq \xi \leq c_{6} \Gamma_{s, r}(d(x)) \quad \text { in } \Omega .
$$

Let $\delta_{2}=\min \left\{1, m_{1}^{r /(1+s)}, \frac{1}{c_{6}}\right\}$. Then

$$
\Delta\left(\delta_{2} \xi\right)-\beta\left(\delta_{2} \xi\right)+m_{1}^{r} \varphi_{1}^{r}\left(\delta_{2} \xi\right)^{-s} \geq \delta_{2}\left(\Delta \xi-\beta \xi+\varphi_{1}^{r} \xi^{-s}\right)=0 \quad \text { in } \Omega,
$$


and by (4.9) we have

$$
\delta_{2} \xi \leq \delta_{2} c_{6} \Gamma_{s, r}(d(x)) \leq \Gamma_{s, r}(\varepsilon) \quad \text { on } \partial \Omega_{\varepsilon} .
$$

Therefore, from (4.6), (4.10) and (4.11) we have obtained

$$
\begin{gathered}
\Delta(T v)-\beta(T v)+m_{1}^{r} \varphi_{1}^{r}(T v)^{-s} \leq 0 \leq \Delta\left(\delta_{2} \xi\right)-\beta\left(\delta_{2} \xi\right)+m_{1}^{r} \varphi_{1}^{r}\left(\delta_{2} \xi\right)^{-s} \quad \text { in } \Omega, \\
T v=\Gamma_{s, r}(\varepsilon) \geq \delta_{2} \xi \quad \text { on } \partial \Omega_{\varepsilon} .
\end{gathered}
$$

By Lemma 3.3 it follows that $T v \geq \delta_{2} \xi$ in $\Omega_{\varepsilon}$. In view of (4.8), the last inequality leads us to $T v \geq \delta_{2} c_{3} \Gamma_{s, r}\left(\varphi_{1}\right)$ in $\Omega_{\varepsilon}$. Thus, we consider

$$
m_{2}=\min \left\{1, \delta_{2} c_{3}\right\}>0
$$

in the definition of the set $\mathcal{A}_{\varepsilon}$. Note that $m_{2}$ is independent of $\varepsilon$ and $T v \geq$ $m_{2} \Gamma_{s, r}\left(\varphi_{1}\right)$ in $\Omega_{\varepsilon}$.

The definition of $\mathcal{A}_{\varepsilon}$ and (3.19) yield

$$
v \geq m_{2} \Gamma_{s, r}\left(\varphi_{1}\right) \geq m_{2} \varphi_{1}^{\sigma} \quad \text { in } \Omega_{\varepsilon} .
$$

Using the estimate $v \geq m_{2} \varphi_{1}^{\sigma}$ in the first equation of (4.4) we get

$$
\Delta(T u)-\alpha(T u)+m_{2}^{-q} \varphi_{1}^{-q \sigma}(T u)^{p}+\rho(x) \geq 0 \text { in } \Omega_{\varepsilon} .
$$

As above, we next consider the problem

$$
\begin{cases}\Delta \zeta-\alpha \zeta+m_{2}^{-q} \varphi_{1}^{-q \sigma} \zeta^{p}+\rho(x)=0 & \text { in } \Omega, \\ \zeta>0 & \text { in } \Omega, \\ \zeta=0 & \text { on } \partial \Omega .\end{cases}
$$

Since $q \sigma<p+1$, by Proposition 3.4 there exists $\zeta \in C^{2}(\Omega) \cap C(\bar{\Omega})$, a unique solution of (4.13), such that

$$
c_{7} \varphi_{1} \leq \zeta \leq c_{8} \varphi_{1} \text { in } \Omega,
$$

for some $c_{7}, c_{8}>0$. Note that $q \sigma<p+1$, (4.14) and Lemma 3.1)imply $\Delta \zeta \in L^{1}(\Omega)$. Let $A_{1}=\max \left\{1, \frac{1}{C c_{7}}\right\}$. Then

$$
\Delta\left(A_{1} \zeta\right)-\alpha\left(A_{1} \zeta\right)+m_{2}^{-q} \varphi_{1}^{-q \sigma}\left(A_{1} \zeta\right)^{p}+\rho(x) \leq 0 \quad \text { in } \Omega_{\varepsilon} .
$$

Also by (3.1) and (4.14) we have

$$
A_{1} \zeta \geq A_{1} c_{7} \varphi_{1} \geq A_{1} C c_{7} d(x) \geq \varepsilon \quad \text { on } \partial \Omega_{\varepsilon} .
$$

Define

$$
\Psi(x, t)=-\alpha t+m_{2}^{-q} \varphi_{1}^{-q \sigma}(x)\left(A_{1} t\right)^{p}+\rho(x), \quad(x, t) \in \Omega_{\varepsilon} \times(0, \infty) .
$$

Then $\Psi$ satisfies the hypotheses in Lemma 3.2 and

$$
\begin{gathered}
\Delta\left(A_{1} \zeta\right)+\Psi\left(x, A_{1} \zeta\right) \leq 0 \leq \Delta(T u)+\Psi(x, T u) \quad \text { in } \Omega_{\varepsilon}, \\
T u, A_{1} \zeta>0 \text { in } \Omega_{\varepsilon}, T u=\varepsilon \leq A_{1} \zeta \text { on } \Omega_{\varepsilon}, \\
\Delta\left(A_{1} \zeta\right) \in L^{1}\left(\Omega_{\varepsilon}\right) .
\end{gathered}
$$

By Lemma 3.2 it follows that $T u \leq A_{1} \zeta$ in $\Omega_{\varepsilon}$. In view of (4.14), let us take $M_{1}:=\max \left\{1, A_{1} c_{8}\right\}$ in the definition of the set $\mathcal{A}_{\varepsilon}$. Then $M_{1}$ does not depend on $\varepsilon$, and by (4.14) we have

$$
T u \leq M_{1} \varphi_{1} \quad \text { in } \Omega_{\varepsilon} .
$$


The definition of $\mathcal{A}_{\varepsilon}$ yields $u \leq M_{1} \varphi_{1}$ in $\Omega_{\varepsilon}$. Then, the second equation of system (4.4) produces

$$
\Delta(T v)-\beta(T v)+\frac{M_{1}^{r} \varphi_{1}^{r}}{(T v)^{s}} \geq 0 \quad \text { in } \Omega_{\varepsilon} .
$$

Let $A_{2}=\max \left\{1, M_{1}^{r}, \frac{1}{c_{5}}\right\}$. If $\xi$ is the unique solution of (4.7), then

$$
\Delta\left(A_{2} \xi\right)-\beta\left(A_{2} \xi\right)+M_{1}^{r} \varphi_{1}^{r}\left(A_{2} \xi\right)^{-s} \leq 0 \quad \text { in } \Omega_{\varepsilon},
$$

and by (4.9) we also have

$$
A_{2} \xi \geq A_{2} c_{5} \Gamma_{s, r}(d(x)) \geq \Gamma_{s, r}(\varepsilon) \quad \text { on } \partial \Omega_{\varepsilon} .
$$

Therefore, by Lemma 3.3 it follows that $T v \leq A_{2} \xi$ in $\Omega_{\varepsilon}$. Now, we take $M_{2}:=$ $\max \left\{1, A_{2} c_{4}\right\}$ in the definition of the set $\mathcal{A}_{\varepsilon}$. It follows that $M_{2}$ is independent of $\varepsilon$ and, by virtue of (4.8), we obtain $T v \leq M_{2} \Gamma_{s, r}\left(\varphi_{1}\right)$ in $\Omega_{\varepsilon}$. This finishes the proof of our Lemma 4.1 .

Lemma 4.2. The mapping $\mathcal{T}: \mathcal{A}_{\varepsilon} \rightarrow \mathcal{A}_{\varepsilon}$ defined in (4.3)-(4.4) is compact and continuous.

Proof. Let us fix $(u, v) \in \mathcal{A}_{\varepsilon}$. Then $u, v, T u$ and $T v$ are bounded away from zero in $\bar{\Omega}_{\varepsilon}$, which yields

$$
\left\|\frac{(T u)^{p}}{v^{q}}\right\|_{L^{\infty}\left(\Omega_{\varepsilon}\right)},\left\|\frac{u^{r}}{(T v)^{s}}\right\|_{L^{\infty}\left(\Omega_{\varepsilon}\right)} \leq c_{\varepsilon}=c\left(\varepsilon, m_{1}, m_{2}, M_{1} \cdot M_{2}, p, q, r, s\right) .
$$

Hence, by Hölder estimates, for all $\tau>N$ we obtain

$$
\|T u\|_{W^{2, \tau}\left(\Omega_{\varepsilon}\right)},\|T v\|_{W^{2, \tau}\left(\Omega_{\varepsilon}\right)} \leq c_{1, \varepsilon},
$$

for some $c_{1, \varepsilon}>0$ independent of $u$ and $v$. Since the embedding $W^{2, \tau}\left(\Omega_{\varepsilon}\right) \hookrightarrow$ $C^{1, \gamma}\left(\bar{\Omega}_{\varepsilon}\right), 0<\gamma<1-N / \tau$, is compact, we derive that the mapping $\mathcal{T}: \mathcal{A}_{\varepsilon} \rightarrow$ $\mathcal{A}_{\varepsilon} \subset C\left(\bar{\Omega}_{\varepsilon}\right) \times C\left(\bar{\Omega}_{\varepsilon}\right)$ is also compact.

It remains to prove that $\mathcal{T}$ is continuous. To this aim, let $\left\{\left(u_{n}, v_{n}\right)\right\}_{n \geq 1} \subset \mathcal{A}_{\varepsilon}$ be such that $u_{n} \rightarrow u$ and $v_{n} \rightarrow v$ in $C\left(\bar{\Omega}_{\varepsilon}\right)$ as $n \rightarrow \infty$. Since $\mathcal{T}$ is compact, there exists $(U, V) \in \mathcal{A}_{\varepsilon}$ such that up to a subsequence we have

$$
\mathcal{T}\left(u_{n}, v_{n}\right) \rightarrow(U, V) \quad \text { in } \mathcal{A}_{\varepsilon} \text { as } n \rightarrow \infty .
$$

Using the $L^{\infty}\left(\Omega_{\varepsilon}\right)$ bounds of $\left(\frac{\left(T u_{n}\right)^{p}}{v_{n}^{q}}\right)_{n \geq 1}$ and $\left(\frac{u_{n}^{r}}{\left(T v_{n}\right)^{s}}\right)_{n \geq 1}$, it follows that $\left(T u_{n}\right)_{n \geq 1}$ and $\left(T v_{n}\right)_{n \geq 1}$ are bounded in $W^{2, \tau}\left(\Omega_{\varepsilon}\right)$ for all $\tau>N$. As before, this implies that $\left(T u_{n}\right)_{n \geq 1}$ and $\left(T v_{n}\right)_{n \geq 1}$ are bounded in $C^{1, \gamma}\left(\bar{\Omega}_{\varepsilon}\right)(0<\gamma<1-N / \tau)$. Next, by Schauder estimates, it follows that $\left(T u_{n}\right)_{n \geq 1}$ and $\left(T v_{n}\right)_{n \geq 1}$ are bounded in $C^{2, \gamma}\left(\bar{\Omega}_{\varepsilon}\right)$. Since $C^{2, \gamma}\left(\bar{\Omega}_{\varepsilon}\right)$ is compactly embedded in $C^{2}\left(\bar{\Omega}_{\varepsilon}\right)$, we deduce that up to a subsequence, we have that

$$
T u_{n} \rightarrow U \text { and } T v_{n} \rightarrow V \quad \text { in } C^{2}\left(\bar{\Omega}_{\varepsilon}\right) \text { as } n \rightarrow \infty .
$$

Passing to the limit in (4.4), we get that $(U, V)$ satisfies

$$
\begin{cases}\Delta U-\alpha U+\frac{U^{p}}{v^{q}}+\rho(x)=0, U>0, & \text { in } \Omega_{\varepsilon}, \\ \Delta V-\beta V+\frac{u^{r}}{V^{s}}=0, V>0, & \text { in } \Omega_{\varepsilon}, \\ U=\varepsilon, \quad V=\Gamma_{s, r}(\varepsilon) & \text { on } \partial \Omega_{\varepsilon} .\end{cases}
$$


Using the uniqueness of (4.4), it follows that $T u=U$ and $T v=V$. Thus, we have obtained that any subsequence of $\left\{\mathcal{T}\left(u_{n}, v_{n}\right)\right\}_{n \geq 1}$ has a subsequence converging to $\mathcal{T}(u, v)$ in $\mathcal{A}_{\varepsilon}$. But this implies that the entire sequence $\left\{\mathcal{T}\left(u_{n}, v_{n}\right)\right\}_{n \geq 1}$ converges to $\mathcal{T}(u, v)$ in $\mathcal{A}_{\varepsilon}$, whence the continuity of $\mathcal{T}$. The proof of Lemma 4.2 is now complete.

Proof of Theorem 2.1 completed. According to Lemmas 4.1 and 4.2 we are now in a position to apply Schauder's fixed point theorem. Thus, for all $0<\varepsilon<\varepsilon_{0}$, there exists $\left(u_{\varepsilon}, v_{\varepsilon}\right) \in \mathcal{A}_{\varepsilon}$ such that $\mathcal{T}\left(u_{\varepsilon}, v_{\varepsilon}\right)=\left(u_{\varepsilon}, v_{\varepsilon}\right)$. By standard elliptic regularity arguments, we deduce $u_{\varepsilon}, v_{\varepsilon} \in C^{2}\left(\bar{\Omega}_{\varepsilon}\right)$. Therefore, for all $0<\varepsilon<\varepsilon_{0}$ we have proved the existence of a solution $\left(u_{\varepsilon}, v_{\varepsilon}\right) \in C^{2}\left(\bar{\Omega}_{\varepsilon}\right) \times C^{2}\left(\bar{\Omega}_{\varepsilon}\right)$ of system (4.2). Next, we extend $u_{\varepsilon}=\varepsilon, v_{\varepsilon}=\Gamma_{s, r}(\varepsilon)$ in $\Omega \backslash \bar{\Omega}_{\varepsilon}$. Furthermore, by the definition of $\mathcal{A}_{\varepsilon}$ we have

$$
\begin{aligned}
m_{1} \varphi_{1} & \leq u_{\varepsilon} \leq M_{1} \varphi_{1}+\varepsilon \leq M_{1} \varphi_{1}+\varepsilon_{0} \quad \text { in } \Omega, \\
m_{2} \Gamma_{s, r}\left(\varphi_{1}\right) & \leq v_{\varepsilon} \leq M_{2} \Gamma_{s, r}\left(\varphi_{1}\right)+\Gamma_{s, r}(\varepsilon) \leq M_{1} \varphi_{1}+c_{\varepsilon_{0}} \quad \text { in } \Omega .
\end{aligned}
$$

As above, $L^{\infty}$ bounds together with Hölder estimates yield $\left(u_{\varepsilon}\right)_{0<\varepsilon<\varepsilon_{0}},\left(v_{\varepsilon}\right)_{0<\varepsilon<\varepsilon_{0}}$, bounded in $W_{l o c}^{2, \tau}(\Omega)$, for all $\tau>N$. With similar arguments, there exist $u, v \in$ $C^{2}(\Omega)$ such that for all $\omega \subset \subset \Omega,\left(u_{\varepsilon}\right)_{0<\varepsilon<\varepsilon_{0}}$ and $\left(v_{\varepsilon}\right)_{0<\varepsilon<\varepsilon_{0}}$ converge up to a subsequence to $u$ and $v$ respectively in $C^{2}(\bar{\omega})$ as $\varepsilon \rightarrow 0$. Passing to the limit with $\varepsilon \rightarrow 0$ in (4.2) and (4.16)-(4.17) we get

$$
\begin{cases}\Delta u-\alpha u+\frac{u^{p}}{v^{q}}+\rho(x)=0 & \text { in } \Omega, \\ \Delta v-\beta v+\frac{u^{r}}{v^{s}}=0 & \text { in } \Omega,\end{cases}
$$

and

$$
\begin{aligned}
m_{1} \varphi_{1} & \leq u \leq M_{1} \varphi_{1} \quad \text { in } \Omega, \\
m_{2} \Gamma_{s, r}\left(\varphi_{1}\right) & \leq v \leq M_{2} \Gamma_{s, r}\left(\varphi_{1}\right) \quad \text { in } \Omega .
\end{aligned}
$$

Now, we extend $u=v=0$ on $\partial \Omega$. From (4.18) and (4.19) we deduce that $u, v \in$ $C(\bar{\Omega})$. Hence, the system (1.3) has a classical solution $(u, v)$.

It remains to establish the boundary estimates of the solution to (1.3). This follows essentially by using the same arguments as above. Let $(u, v)$ be an arbitrary solution of (1.3). Then $\Delta u-\alpha u+\rho(x) \leq 0$ in $\Omega$ which implies that $u \geq w$ in $\Omega$, where $w$ is the unique solution of (3.5). By (3.6) it follows that $u \geq c_{1} \varphi_{1}$ in $\Omega$. Using this inequality in the second equation of (1.3) we deduce $\Delta v-\beta v+c_{2} \varphi_{1}^{r} v^{-s} \leq 0$ in $\Omega$ for some $c_{2}>0$ (we actually have $c_{2}=c_{1}^{r}>0$ ). Next, let $\xi$ be the unique solution of (4.7). A similar argument to that used in Lemma 4.1 yields $v \geq c_{3} \xi$ in $\Omega$. In view of estimate (3.21) in Proposition 3.5 we derive that $v \geq c_{4} \Gamma_{s, r}\left(\varphi_{1}\right)$ in $\Omega$ for some $c_{4}>0$. According to (3.19) it follows that $v \geq c_{5} \varphi_{1}^{\sigma}$ in $\Omega$. This inequality combined with the first equation in system (1.3) produces $\Delta u-\alpha u+c_{6} \varphi_{1}^{-q \sigma} u^{p}+\rho(x) \geq 0$ in $\Omega$.

Consider the problem

$$
\begin{cases}\Delta z-\alpha z+c_{6} \varphi_{1}^{-q \sigma} z^{p}+\rho(x)=0 & \text { in } \Omega, \\ z>0 & \text { in } \Omega, \\ z=0 & \text { on } \partial \Omega .\end{cases}
$$


Since $q \sigma<p+1$, by Proposition 3.4 there exists a unique solution of (4.20) such that $z \leq c_{7} \varphi_{1}$ in $\Omega$. Thus, by Lemma 3.2 we get $u \leq z \leq c_{7} \varphi_{1}$ in $\Omega$. Using this last inequality in the second equation of (1.3) we finally obtain $\Delta v-\beta v+c_{8} \varphi_{1}^{r} v^{-s} \geq 0$ in $\Omega$ for some $c_{8}>0$. By virtue of Proposition 3.5 we have $v \leq c_{9} \Gamma_{s, r}\left(\varphi_{1}\right)$ in $\Omega$. Thus, we have obtained

$$
\begin{aligned}
m_{1} \varphi_{1} & \leq u \leq m_{2} \varphi_{1} \quad \text { in } \Omega, \\
m_{1} \Gamma_{s, r}\left(\varphi_{1}\right) & \leq u \leq m_{2} \Gamma_{s, r}\left(\varphi_{1}\right) \quad \text { in } \Omega
\end{aligned}
$$

for some fixed constants $m_{1}, m_{2}>0$. Now, the boundary estimates in Theorem 2.1 follow from the above inequalities combined with (3.1). This concludes the proof.

4.2. Proof of Corollary 2.2. Let $(u, v)$ be a classical solution of (1.3). We rewrite the system (1.3) in the form

$$
\Delta u=f_{1}(x) \text { in } \Omega, \quad \Delta v=f_{2}(x) \text { in } \Omega, \quad u=v=0 \text { on } \partial \Omega,
$$

where

$$
f_{1}(x)=\alpha u(x)-\frac{u^{p}(x)}{v^{q}(x)}-\rho(x), f_{2}(x)=\beta v(x)-\frac{u^{r}(x)}{v^{s}(x)}, \text { for all } x \in \Omega .
$$

Note that in our settings we have $\sigma=1$ in (2.2), and by virtue of Theorem 2.1 there exist $c_{1}, c_{2}>0$ such that $c_{1} d(x) \leq u, v \leq c_{2} d(x)$ in $\Omega$. Hence,

$$
\left|f_{1}(x)\right| \leq m_{1} d^{p-q}(x)+\rho(x) \quad \text { in } \Omega \quad \text { and } \quad\left|f_{2}(x)\right| \leq m_{2} d^{r-s}(x) \quad \text { in } \Omega .
$$

(i) Since $0 \leq p-q$ and $0 \leq r-s$, by (4.21) we get $f_{1}, f_{2} \in L^{\infty}(\Omega)$. Next, standard elliptic arguments lead us to $u, v \in C^{2}(\bar{\Omega})$.

(ii) Let us assume that $-1<p-q<0$ and $-1<r-s<0$. From (4.21) we derive

$$
\left|f_{1}(x)\right| \leq c d^{p-q}(x) \quad \text { in } \Omega \quad \text { and } \quad\left|f_{2}(x)\right| \leq c d^{r-s}(x) \quad \text { in } \Omega,
$$

for some positive constant $c>0$.

If $N=1$, then for all $x_{1}, x_{2} \in \Omega$ we have

$$
\left|u^{\prime}\left(x_{1}\right)-u^{\prime}\left(x_{2}\right)\right| \leq\left|\int_{x_{1}}^{x_{2}}\right| f_{1}(t)|d t| \leq c\left|\int_{x_{1}}^{x_{2}} d^{p-q}(t) d t\right| \leq \tilde{c}\left|x_{1}-x_{2}\right|^{1+p-q},
$$

where $\tilde{c}>0$ does not depend on $x_{1}, x_{2}$. This yields $u \in C^{1,1+p-q}(\bar{\Omega})$ and similarly $v \in C^{1,1+r-s}(\bar{\Omega})$.

If $N \geq 2$, the conclusion follows exactly in the same way as in 9 . More precisely, let $\mathcal{G}$ denote the Green's function for the Laplace operator. Then for all $x \in \Omega$ we have

$$
u(x)=\int_{\Omega} \mathcal{G}(x, y) f_{1}(y) d y, \quad v(x)=\int_{\Omega} \mathcal{G}(x, y) f_{2}(y) d y
$$

and

$$
\nabla u(x)=\int_{\Omega} \mathcal{G}_{x}(x, y) f_{1}(y) d y, \quad \nabla v(x)=\int_{\Omega} \mathcal{G}_{x}(x, y) f_{2}(y) d y
$$


Then, for all $x_{1}, x_{2} \in \Omega, x_{1} \neq x_{2}$ we have

$$
\begin{aligned}
\left|\nabla u\left(x_{1}\right)-\nabla u\left(x_{2}\right)\right| & \leq \int_{\Omega}\left|\mathcal{G}_{x}\left(x_{1}, y\right)-\mathcal{G}_{x}\left(x_{2}, y\right)\right|\left|f_{1}(y)\right| d y \\
& \leq c \int_{\Omega}\left|\mathcal{G}_{x}\left(x_{1}, y\right)-\mathcal{G}_{x}\left(x_{2}, y\right)\right| d^{p-q}(y) d y,
\end{aligned}
$$

and similarly

$$
\left|\nabla v\left(x_{1}\right)-\nabla v\left(x_{2}\right)\right| \leq c \int_{\Omega}\left|\mathcal{G}_{x}\left(x_{1}, y\right)-\mathcal{G}_{x}\left(x_{2}, y\right)\right| d^{r-s}(y) d y .
$$

From now on, we need only to employ the sharp estimates given in [9, Theorem 1.1] in order to obtain $u \in C^{1,1+p-q}(\bar{\Omega})$ and $v \in C^{1,1+r-s}(\bar{\Omega})$. This finishes the proof of Corollary 2.2.

4.3. Proof of Theorem 2.3, Let $(u, v)$ be a classical solution of (2.3). Then, by virtue of Corollary [2.2, we have

$$
u, v \in C^{2}[0,1] \times C^{2}[0,1] \quad \text { if } 0 \leq p-q, 0 \leq r-s,
$$

and

$$
u \in C^{2}(0,1) \cap C^{1,1+p-q}[0,1], v \in C^{2}(0,1) \cap C^{1,1+r-s}[0,1],
$$

if $-1<p-q<0,-1<r-s<0$. Furthermore, by Hopf's maximum principle we also have that $u^{\prime}(0)>0, v^{\prime}(0)>0, u^{\prime}(1)<0$ and $v^{\prime}(1)<0$.

Assume that there exist $\left(u_{1}, v_{1}\right)$ and $\left(u_{2}, v_{2}\right)$, two different solutions of (2.3).

First we claim that we cannot have $u_{2} \geq u_{1}$ or $v_{2} \geq v_{1}$ in $[0,1]$. Assume by contradiction that $u_{2} \geq u_{1}$ in $[0,1]$. Then

$$
v_{2}^{\prime \prime}-\beta v_{2}+\frac{u_{2}^{r}}{v_{2}^{s}}=0=v_{1}^{\prime \prime}-\beta v_{1}+\frac{u_{1}^{r}}{v_{1}^{s}} \quad \text { in }(0,1),
$$

and by Lemma 3.3 we get $v_{2} \geq v_{1}$ in $[0,1]$. This implies that

$$
u_{1}^{\prime \prime}-\alpha u_{1}+\frac{u_{1}^{p}}{v_{2}^{q}}+\rho(x) \leq 0=u_{2}^{\prime \prime}-\alpha u_{2}+\frac{u_{2}^{p}}{v_{2}^{q}}+\rho(x) \quad \text { in }(0,1) .
$$

On the other hand, the mapping $\Psi(x, t)=-\alpha t+\frac{t^{p}}{v_{2}^{q}(x)}+\rho(x),(x, t) \in(0,1) \times$ $(0, \infty)$ satisfies the hypotheses in Lemma 3.2. Hence $u_{2} \leq u_{1}$ in $[0,1]$; that is, $u_{1} \equiv u_{2}$. This also implies $v_{1} \equiv v_{2}$, which is a contradiction. Replacing $u_{1}$ by $u_{2}$ and $v_{1}$ by $v_{2}$, we also get that the situation $u_{1} \geq u_{2}$ or $v_{1} \geq v_{2}$ in $[0,1]$ is not possible.

Set $U=u_{2}-u_{1}$ and $V=v_{2}-v_{1}$. From the above arguments, both $U$ and $V$ change sign in $(0,1)$. The key result in our approach is the following.

Proposition 4.3. $U$ and $V$ vanish only at finitely many points in the interval $[0,1]$.

Proof. Subtracting the corresponding equations for $\left(u_{1}, v_{1}\right)$ and $\left(u_{2}, v_{2}\right)$ we obtain the following linear problem:

$$
\left\{\begin{array}{l}
\mathbf{W}^{\prime \prime}(x)+A(x) \mathbf{W}(x)=\mathbf{0} \\
\mathbf{W}(0)=\mathbf{W}(1)=\mathbf{0},
\end{array} \quad \text { in }(0,1),\right.
$$


where $\mathbf{W}=(U, V)^{T}$ and $A(x)=\left(A_{i j}(x)\right)_{1 \leq i, j \leq 2}$ is a $2 \times 2$ matrix defined as

$$
\begin{aligned}
& A_{11}(x)=-\alpha+\left\{\begin{array}{cc}
\frac{1}{v_{2}^{q}(x)} \cdot \frac{u_{2}^{p}(x)-u_{1}^{p}(x)}{u_{2}(x)-u_{1}(x)}, & u_{1}(x) \neq u_{2}(x), \\
p \frac{u_{1}^{p-1}(x)}{v_{1}^{q}(x)}, & u_{1}(x)=u_{2}(x),
\end{array}\right. \\
& A_{12}(x)=\left\{\begin{array}{cc}
-\frac{u_{1}^{p}(x)}{v_{1}^{q}(x) v_{2}^{q}(x)} \cdot \frac{v_{2}^{q}(x)-v_{1}^{q}(x)}{v_{2}(x)-v_{1}(x)}, & v_{1}(x) \neq v_{2}(x), \\
-q \frac{u_{1}^{p}(x)}{v_{1}^{q+1}(x)}, & v_{1}(x)=v_{2}(x),
\end{array}\right. \\
& A_{21}(x)=\left\{\begin{array}{cc}
\frac{1}{v_{2}^{s}(x)} \cdot \frac{u_{2}^{r}(x)-u_{1}^{r}(x)}{u_{2}(x)-u_{1}(x)}, & u_{1}(x) \neq u_{2}(x), \\
r \frac{u_{1}^{r-1}(x)}{v_{1}^{s}(x)}, & u_{1}(x)=u_{2}(x),
\end{array}\right. \\
& A_{22}(x)=-\beta-\left\{\begin{array}{cc}
\frac{u_{1}^{r}(x)}{v_{1}^{s}(x) v_{2}^{s}(x)} \cdot \frac{v_{2}^{s}(x)-v_{1}^{s}(x)}{v_{2}(x)-v_{1}(x)}, & v_{1}(x) \neq v_{2}(x), \\
s \frac{u_{1}^{r}(x)}{v_{1}^{s+1}(x)}, & v_{1}(x)=v_{2}(x) .
\end{array}\right.
\end{aligned}
$$

Lemma 4.4. We have

(i) $A_{i j} \in C(0,1)$, for all $1 \leq i, j \leq 2$;

(ii) $A_{12}(x) \neq 0$ and $A_{21}(x) \neq 0$ for all $x \in(0,1)$;

(iii) $d^{1-(p-q)}(x) A_{1 j} \in L^{\infty}(0,1)$ and $d^{1-(r-s)}(x) A_{2 j} \in L^{\infty}(0,1)$, for $j=1,2$.

Proof. The claims in (i) and (ii) are easy to verify. We only prove the statement in (iii). To this aim, let us notice first that by the regularity of solutions, there exist $c_{1}, c_{2}>0$ such that

$$
c_{1} d(x) \leq u_{i}, v_{i} \leq c_{2} d(x) \quad \text { in }(0,1), 1 \leq i \leq 2 .
$$

By (4.24) and the fact that

$$
\left|a^{q}-b^{q}\right| \leq q|a-b| \max \left\{a^{q-1}, b^{q-1}\right\} \quad \text { for all } a, b>0,
$$

we have

$$
\begin{aligned}
d(x)\left|A_{12}(x)\right| & \leq q d(x) \frac{u_{1}^{p}(x)}{v_{1}^{q}(x) v_{2}^{q}(x)} \max \left\{v_{1}^{q-1}(x), v_{2}^{q-1}(x)\right\} \\
& \leq q d^{p-q}(x)\left(\frac{u_{1}(x)}{d(x)}\right)^{p} \max \left\{\left(\frac{d(x)}{v_{1}(x)}\right)^{q+1},\left(\frac{d(x)}{v_{2}(x)}\right)^{q+1}\right\} \\
& \leq c d^{p-q}(x) \text { for all } 0<x<1 .
\end{aligned}
$$

Hence $d^{1-(p-q)}(x) A_{12} \in L^{\infty}(0,1)$. We obtain similar estimates for $d^{1-(p-q)}(x) A_{11}$ and $d^{1-(r-s)}(x) A_{2 j}, j=1,2$. This concludes the proof.

Next, Lemma 4.4(i)-(ii) allows us to employ the following result which is proved in [1, Lemma 7].

Lemma 4.5 (see [1]). Let $0<a<b<1$ and $A=\left(A_{i j}\right)_{1 \leq i, j \leq 2}$ be such that

(i) $A_{i j} \in C[a, b]$, for all $1 \leq i, j \leq 2$;

(ii) $A_{12}(x) \neq 0$ and $A_{21}(x) \neq 0$ for all $x \in[a, b]$. 
Assume that there exists $\mathbf{W}=(U, V)^{T} \in C^{2}[a, b] \times C^{2}[a, b]$ such that $\mathbf{W} \not \equiv 0$ and $\mathbf{W}^{\prime \prime}(x)+A(x) \mathbf{W}(x)=\mathbf{0}$ in $[a, b]$. Then, neither $U$ nor $V$ can have infinitely many zeros in $[a, b]$.

As a consequence, we deduce that if $\mathbf{W}$ is a solution of (4.23) and $\mathbf{W}$ vanishes for infinitely many times in an interval $[a, b] \subset(0,1)$, then, applying Lemma 4.5 in $[\varepsilon, 1-\varepsilon]$ for all $\varepsilon>0$ sufficiently small, we get $\mathbf{W} \equiv \mathbf{0}$.

It remains to show that $U$ and $V$ cannot vanish infinitely many times in the neighborhood of $x=0$ and $x=1$. We shall consider only the case $x=0$; the situation where $U$ or $V$ have infinitely many zeros near $x=1$ can be handled in the same manner.

Without loss of generality, we may assume that $V$ has infinitely many zeros in a neighborhood of $x=0$. By the continuity of $V$ it follows that $V(0)=0$. Furthermore, since $V \in C^{2}(0,1) \cap C^{1}[0,1]$, by Rolle's Theorem we get that both $V^{\prime}$ and $V^{\prime \prime}$ have infinitely many zeros near $x=0$. Therefore, $V^{\prime}(0)=0$; that is, $v_{1}^{\prime}(0)=v_{2}^{\prime}(0)$.

If $U^{\prime}(0)=0$, then $\mathbf{W}(0)=\mathbf{W}^{\prime}(0)=\mathbf{0}$. Let $\gamma=\min \{0, p-q, r-s\}$. Then $-1<\gamma \leq 0$, and by Lemma 4.4(iii) it follows that $x^{1-\gamma} A_{i j} \in L^{\infty}(0,1 / 2)$. Thus, we can use Proposition 5.3 in the Appendix in order to get that $\mathbf{W} \equiv \mathbf{0}$ in $[0,1 / 2]$. Then, by Lemma 4.5 we obtain $\mathbf{W} \equiv 0$ in $[0,1]$, which is a contradiction. Hence $U^{\prime}(0) \neq 0$. Subtracting the second equation corresponding to $v_{1}$ and $v_{2}$ in the system (2.3) we have

$$
\begin{aligned}
V^{\prime \prime}(x) & =\beta V(x)+\frac{u_{1}^{r}(x)}{v_{1}^{s}(x)}-\frac{u_{2}^{r}(x)}{v_{2}^{s}(x)} \\
& =x^{r-s}\left\{\beta \frac{V(x)}{x^{r-s}}+\left(\frac{u_{1}(x)}{x}\right)^{r}\left(\frac{x}{v_{1}(x)}\right)^{s}-\left(\frac{u_{2}(x)}{x}\right)^{r}\left(\frac{x}{v_{2}(x)}\right)^{s}\right\} .
\end{aligned}
$$

Since $r-s<1, v_{1}^{\prime}(0)=v_{2}^{\prime}(0)>0$ and $u_{1}^{\prime}(0) \neq u_{2}^{\prime}(0)$, we get

$$
\begin{aligned}
\lim _{x \rightarrow 0^{+}} & \left\{\beta \frac{V(x)}{x^{r-s}}+\left(\frac{u_{1}(x)}{x}\right)^{r}\left(\frac{x}{v_{1}(x)}\right)^{s}-\left(\frac{u_{2}(x)}{x}\right)^{r}\left(\frac{x}{v_{2}(x)}\right)^{s}\right\} \\
& =\frac{u_{1}^{\prime r}(0)-u_{2}^{\prime r}(0)}{v_{1}^{\prime s}(0)} \neq 0 .
\end{aligned}
$$

From (4.25) and (4.26) we derive that $V^{\prime \prime}$ has constant sign in a small neighborhood of $x=0$, which contradicts the fact that $V$ vanishes for infinitely many times in the neighborhood of $x=0$. This finishes the proof of Proposition 4.3.

Proof of Theorem 2.3 completed. Let us define

$$
\begin{array}{ll}
\mathcal{I}^{+}=\{x \in[0,1]: U(x) \geq 0\}, & \mathcal{I}^{-}=\{x \in[0,1]: U(x) \leq 0\}, \\
\mathcal{J}^{+}=\{x \in[0,1]: V(x) \geq 0\}, & \mathcal{J}^{-}=\{x \in[0,1]: V(x) \leq 0\} .
\end{array}
$$

Since both $U$ and $V$ have a finite number of zeros, it follows that the above sets consist of finitely many disjoint closed intervals. Therefore, $\mathcal{I}^{+}=\bigcup_{i=1}^{m} I_{i}^{+}$. For our convenience, let $I^{+}$denote any interval $I_{i}^{+}$, and similar notation will be used for $I^{-}, J^{+}$and $J^{-}$. We have

Lemma 4.6. For all intervals $I^{+}, I^{-}, J^{+}$and $J^{-}$defined above, the following situations cannot occur:
(i) $I^{+} \subset J^{+}$;
(ii) $I^{-} \subset J^{-}$;
(iii) $J^{+} \subset I^{-}$;
(iv) $J^{-} \subset I^{+}$. 
Proof. (i) Assume by contradiction that $I^{+} \subset J^{+}$. This yields $u_{2} \geq u_{1}$ and $v_{2} \geq v_{1}$ in $I^{+}$. Furthermore we have

$$
\begin{aligned}
& u_{1}^{\prime \prime}-\alpha u_{1}+\frac{u_{1}^{p}}{v_{2}^{q}}+\rho(x) \leq 0=u_{2}^{\prime \prime}-\alpha u_{2}+\frac{u_{2}^{p}}{v_{2}^{q}}+\rho(x) \quad \text { in } I^{+}, \\
& u_{1}, u_{2}>0 \quad \text { in } I^{+}, u_{1}=u_{2}=0 \quad \text { on } \partial I^{+}, \quad u_{1}^{\prime \prime} \in L^{1}(0,1) .
\end{aligned}
$$

Thus, by Lemma 3.2 with $\Psi(x, t)=-\alpha t+\frac{t^{p}}{v_{2}^{q}(x)}+\rho(x),(x, t) \in I^{+} \times(0, \infty)$, it follows that $u_{2} \leq u_{1}$ in $I^{+}$. Since $u_{2} \geq u_{1}$ in $I^{+}$, we deduce $u_{1}=u_{2}$ in $I^{+}$; that is, $U \equiv 0$, which contradicts Proposition 4.3. Replacing $u_{1}, v_{1}$ with $u_{2}, v_{2}$ in the above arguments we deduce the statement (ii).

(iii) Suppose that $J^{+} \subset I^{-}$. Then $v_{2} \geq v_{1}$ and $u_{1} \geq u_{2}$ in $J^{+}$, which yield

$$
\frac{u_{1}^{r}}{v_{1}^{s}} \geq \frac{u_{2}^{r}}{v_{2}^{s}} \quad \text { in } J^{+} \text {. }
$$

Hence $V=v_{2}-v_{1}$ satisfies

$$
\begin{cases}V^{\prime \prime}-\beta V=\frac{u_{1}^{r}}{v_{1}^{s}}-\frac{u_{2}^{r}}{v_{2}^{s}} \geq 0 & \text { in } J^{+}, \\ V=0 & \text { on } \partial J^{+} .\end{cases}
$$

Therefore, by maximum principle, we have $V \leq 0$ in $J^{+}$. Since $V \geq 0$ in $J^{+}$, it follows that $V \equiv 0$ in $J^{+}$, which again contradicts Proposition 4.3. The proof of (iv) follows in a similar way.

From now on, the proof of Theorem 2.3 follows in the same manner as in [1, Theorem 6].

\section{THE CASE $p<0$}

5.1. Proof of Theorem 2.4. Assume that the system (1.3) has a classical solution $(u, v)$ and set $M=\|u\|_{\infty}$. Then $v$ satisfies

$$
\Delta v-\beta v+c_{1} v^{-s} \geq 0 \quad \text { in } \Omega,
$$

where $c_{1}=M^{r}>0$. By Lemma 3.3 we get $v \leq z$, where $z$ is the unique solution of the problem

$$
\begin{cases}\Delta z-\beta z+c_{1} z^{-s}=0 & \text { in } \Omega, \\ z>0 & \text { in } \Omega, \\ z=0 & \text { on } \partial \Omega .\end{cases}
$$

Furthermore, from the estimate (3.21) in Proposition 3.5 (with $r=0$ ) there exists $c_{2}>0$ such that $v \leq z \leq c_{2} \Gamma_{s, 0}\left(\varphi_{1}\right)$ in $\Omega$, which yields

$$
\begin{cases}v \leq c_{2} \varphi_{1} \text { in } \Omega & \text { if } s<1, \\ v \leq c_{2} \varphi_{1}\left(1+\left|\log \varphi_{1}\right|\right)^{1 /(1+s)} \text { in } \Omega & \text { if } s=1, \\ v \leq c_{2} \varphi_{1}^{2 /(1+s)} \text { in } \Omega & \text { if } s>1 .\end{cases}
$$

If $s=1$ and $q>2$, we fix $0<\theta<1$ such that $q \theta \geq 2$. Let us set

$$
k=\left\{\begin{array}{cc}
1, & \text { if } s<1 \\
\theta, & \text { if } s=1 \\
2 /(s+1), & \text { if } s>1
\end{array}\right.
$$


Then $q k \geq 2$, and by (5.1) we get $v \leq c_{3} \varphi_{1}^{k}$ in $\Omega$, for some $c_{3}>0$. Using this inequality in the first equation of (1.3) we deduce $\Delta u-\alpha u+c \varphi_{1}^{-q k} u^{p}+\rho(x) \leq 0$ in $\Omega$, where $c=c_{3}^{-q}$. This means that $u$ is a super-solution of the following problem:

$$
\begin{cases}\Delta z-\alpha z+c \varphi_{1}^{-q k} z^{p}+\rho(x)=0 & \text { in } \Omega \\ z>0 & \text { in } \Omega \\ z=0 & \text { on } \partial \Omega .\end{cases}
$$

Note that $w \in C^{2}(\bar{\Omega})$ defined as the unique solution of (3.5) is a sub-solution of (5.2). By standard maximum principle it is easy to get $u \geq w$ in $\Omega$. Hence, the problem (5.2) has classical solutions, but this contradicts Proposition 3.5(i), since $q k \geq 2$. Therefore, the system (1.3) has no solutions. The proof of Theorem 2.4 is now complete.

5.2. Proof of Theorem 2.5. For all $0<\varepsilon<\varepsilon_{0}$ let $\Omega_{\varepsilon}$ be defined as in (4.1). For $m_{1}<1<M_{1}$ and $m_{2}<1<M_{2}$ we set

$$
\mathcal{B}_{\varepsilon}=\left\{\begin{array}{r}
m_{1} \varphi_{1} \leq u \leq M_{1} \varphi_{1}^{\nu} \text { in } \Omega_{\varepsilon}, \\
(u, v) \in C\left(\bar{\Omega}_{\varepsilon}\right) \times C\left(\bar{\Omega}_{\varepsilon}\right): m_{2} \Gamma_{s, r}\left(\varphi_{1}\right) \leq v \leq M_{2} \varphi_{1}^{\tau} \text { in } \Omega_{\varepsilon}, \\
u=\varepsilon, v=\Gamma_{s, r}(\varepsilon) \text { on } \partial \Omega_{\varepsilon},
\end{array}\right\},
$$

where

$$
\nu=\left\{\begin{array}{cl}
1, & \text { if } q \sigma<1+p, \\
1 / 2, & \text { if } q \sigma=1+p, \\
\frac{2-q \sigma}{1-p}, & \text { if } q \sigma>1+p,
\end{array} \quad \text { and } \tau=\left\{\begin{array}{cl}
1, & \text { if } s<1+r \nu \\
1 / 2, & \text { if } s=1+r \nu \\
\frac{2+r \nu}{1+s}, & \text { if } s>1+r \nu
\end{array}\right.\right.
$$

In order to prove that $\mathcal{B}_{\varepsilon}$ is not empty, we first remark that $\nu \leq 1$. Therefore, we only need to check that

$$
\Gamma_{s, r}(t) \leq c_{0} t^{\tau} \quad \text { for all } 0<t \leq 1,
$$

for some fixed $c_{0}>0$. To this aim we analyze the cases $s<1+r, s=1+r$ and $s>1+r$.

If $s<1+r$, since $\tau \leq 1$ we have $\Gamma_{s, r}(t)=t \leq t^{\tau}$ for all $0<t \leq 1$.

If $s>1+r$, from $\nu \leq 1$ we have $s>1+r \nu$, which implies $\tau=\frac{2+r \nu}{1+s} \leq \frac{2+r}{1+s}$. Hence

$$
\Gamma_{s, r}(t)=t^{(2+r) /(1+s)} \leq t^{(2+r \nu) /(1+s)}=t^{\tau} \quad \text { for all } 0<t \leq 1 .
$$

Finally, if $s=1+r$, then $s \geq 1+r \nu$, which implies $\tau=1 / 2$ or $\tau=\frac{2+r \nu}{1+s}$. In both cases we have $\tau<1$. Then

$$
\Gamma_{s, r}(t)=t(1+|\ln t|)^{1 /(1+s)} \leq c_{0} t^{\tau} \quad \text { for all } 0<t \leq 1
$$

and for some fixed $c_{0}>0$.

Remark 5.1. Since $t^{\theta}(1+|\log t|)^{1 /(s+1)} \rightarrow 0$ as $t \rightarrow 0$, for all $\theta>0$, we could replace the value $1 / 2$ in the definition of $\nu$ and $\tau$ by any number $\theta \in(0,1)$ in the case $q \sigma=p+1$ and $s=1+r \nu$ respectively.

Therefore, for small $0<m_{1}, m_{2}<1$ and for large values of $M_{1}, M_{2}>1$ the set $\mathcal{B}_{\varepsilon}$ is not empty. 
As in the previous section, for all $(u, v) \in \mathcal{B}_{\varepsilon}$ let us denote by $(T u, T v)$ the unique solution of

$$
\begin{cases}\Delta(T u)-\alpha(T u)+\frac{(T u)^{p}}{v_{r}^{q}}+\rho(x)=0, T u>0, & \text { in } \Omega_{\varepsilon}, \\ \Delta(T v)-\beta(T v)+\frac{u^{r}}{(T v)^{s}}=0, T v>0, & \text { in } \Omega_{\varepsilon}, \\ T u=\varepsilon, T v=\Gamma_{s, r}(\varepsilon) & \text { on } \partial \Omega_{\varepsilon} .\end{cases}
$$

In this way we have defined a mapping

$$
\mathcal{T}: \mathcal{B}_{\varepsilon} \rightarrow C\left(\bar{\Omega}_{\varepsilon}\right) \times C\left(\bar{\Omega}_{\varepsilon}\right), \mathcal{T}(u, v)=(T u, T v) .
$$

Now, we proceed as in the proof of Theorem 2.1. The main point is to show that there exist $0<m_{1}, m_{2}<1$ and $M_{1}, M_{2}>1$ which are independent of $\varepsilon$ such that $\mathcal{T}\left(\mathcal{B}_{\varepsilon}\right) \subseteq \mathcal{B}_{\varepsilon}$. This allows us to employ Schauder's fixed point theorem.

Following the proof of Lemma 4.1 we get the existence of $m_{1}, m_{2} \in(0,1)$ which are independent of $\varepsilon$ and such that

$$
T u \geq m_{1} \varphi_{1}, T v \geq m_{2} \Gamma_{s, r}\left(\varphi_{1}\right) \quad \text { in } \Omega_{\varepsilon} .
$$

Since $\Gamma_{s, r}(t) \geq t^{\sigma}$ for all $0<t \leq 1$, the definition of $\mathcal{B}_{\varepsilon}$ yields $v \geq m_{2} \varphi_{1}^{\sigma}$ in $\Omega_{\varepsilon}$. Furthermore, the first equation in (5.5) produces

$$
\Delta(T u)-\alpha(T u)+m_{2}^{-q} \varphi_{1}^{-q \sigma}(T u)^{p}+\rho(x) \geq 0 \quad \text { in } \Omega_{\varepsilon} .
$$

Let $\zeta \in C^{2}(\Omega) \cap C(\bar{\Omega})$ be the unique solution of (4.13). Since $p<0$ and $q \sigma<2$, we shall make use of Proposition 3.5(ii) instead of Proposition 3.4, as we did in the proof of Theorem 2.1. Therefore, there exist $c_{1}, c_{2}>0$ such that

$$
c_{1} \Gamma_{-p,-q \sigma}\left(\varphi_{1}\right) \leq \zeta \leq c_{2} \Gamma_{-p,-q \sigma}\left(\varphi_{1}\right) \quad \text { in } \Omega .
$$

Note that $\Gamma_{-p,-q \sigma}(t) \geq t$ for all $0<t \leq 1$. Hence, by (3.1) and (5.6) we get

$$
\zeta \geq c_{1} \varphi_{1} \geq C c_{1} d(x) \quad \text { in } \Omega .
$$

Let us fix $A>1$ such that $A C c_{1}>1$. Since $p<0$ we find

$$
\begin{gathered}
\Delta(A \zeta)-\alpha(A \zeta)+m_{2}^{-q} \varphi_{1}^{-q \sigma}(A \zeta)^{p}+\rho(x) \leq 0 \quad \text { in } \Omega_{\varepsilon}, \\
A \zeta \geq \varepsilon=T u \quad \text { on } \partial \Omega_{\varepsilon} .
\end{gathered}
$$

In view of Lemma 3.3 we derive $A \zeta \geq T u$ in $\Omega_{\varepsilon}$, and by (5.6) it follows that

$$
T u \leq A c_{2} \Gamma_{-p,-q \sigma}\left(\varphi_{1}\right) \quad \text { in } \Omega_{\varepsilon} .
$$

Note that $\Gamma_{-p,-q \sigma}(t) \leq \tilde{c} t^{\nu}$ for all $0<t \leq 1$ and for some fixed constant $\tilde{c}>0$. Therefore, we can find $M_{1}>1$ sufficiently large such that $T u \leq M_{1} \varphi_{1}^{\nu}$ in $\Omega_{\varepsilon}$.

Using the estimate $u \leq M_{1} \varphi_{1}^{\nu}$ in $\Omega_{\varepsilon}$, from the second equation in (5.5) we deduce

$$
\Delta(T v)-\beta(T v)+M_{1}^{r} \varphi_{1}^{r \nu}(T v)^{-s} \geq 0 \quad \text { in } \Omega_{\varepsilon} .
$$

Since $\nu \leq 1$, we can easily prove that $\Gamma_{s, r}(t) \leq c_{0} \Gamma_{s, r \nu}(t)$, for all $0<t \leq 1$ and for some positive constant $c_{0}$. This implies that

$$
T v=\Gamma_{s, r}(\varepsilon) \leq c_{0} \Gamma_{s, r \nu}(\varepsilon) \quad \text { on } \partial \Omega_{\varepsilon} .
$$

Next, arguments similar to those in the proof of Lemma 4.1 yield $T v \leq c \Gamma_{s, r \nu}\left(\varphi_{1}\right)$ in $\Omega_{\varepsilon}$. It remains to notice that $\Gamma_{s, r \nu}(t) \leq \bar{c} t^{\tau}$ for all $0<t \leq 1$ and for some $\bar{c}>0$. Hence, $T v \leq M_{2} \varphi_{1}^{\tau}$ in $\Omega_{\varepsilon}$ for some $M_{2}>1$ independent of $\varepsilon$. Therefore $\mathcal{T}\left(\mathcal{B}_{\varepsilon}\right) \subseteq \mathcal{B}_{\varepsilon}$. From now on, we proceed exactly in the same way as in the proof of Theorem 2.1. 
Assume next that $q<p+1$ and $s<r+1$. Then, by (2.2) and (5.3) we get $\sigma=\nu=\tau=1$. With the same arguments as in the proof of Theorem 2.1 we get $m_{1} d(x) \leq u, v \leq m_{2} d(x)$ in $\Omega$, for some $m_{1}, m_{2}>0$ and for all solutions $(u, v)$ of (1.3). Then we use the same approach as in Corollary 2.2 in order to get that $u, v \in C^{2}(\Omega) \cap C^{1, \gamma}(\bar{\Omega})$, for some $0<\gamma<1$. This finishes the proof of Theorem 2.5 .

Remark 5.2. The approach used in this paper can be employed to extend the study of system (1.3) to the following class of exponents:

$$
0 \leq p<1,0<q<p+1, r>0,-1<s \leq 0 .
$$

In this sense, we need the smooth variant of Proposition 3.5 concerning the sublinear case $-1<s \leq 0$. Taking into account the fact that $r>0$, if $-1<s \leq 0$, then the problem (3.16) has a unique solution $v \in C^{2}(\bar{\Omega})$. One can show that system (1.3) has classical solutions and that any solution $(u, v)$ of (1.3) satisfies

$$
c_{1} d(x) \leq u, v \leq c_{2} d(x) \quad \text { in } \Omega,
$$

for some $c_{1}, c_{2}>0$. Furthermore, with the same idea as in the proof of Corollary 2.2 we get

(i) if $p \geq q$, then $u, v \in C^{2}(\bar{\Omega})$;

(ii) if $-1<p-q<0$, then $u \in C^{2}(\Omega) \cap C^{1,1+p-q}(\bar{\Omega})$ and $v \in C^{2}(\bar{\Omega})$.

\section{APPENDIX}

In this part we prove a result which generalizes Lemma 8 in [1]. More precisely we have

Proposition 5.3. Let $0<a<1,-1<\gamma \leq 0$ and $A=\left(A_{i j}\right)_{1 \leq i, j \leq 2}$ be $a 2 \times 2$ matrix such that for all $1 \leq i, j \leq 2$ we have

$$
A_{i j} \in C(0, a] \quad \text { and } \quad x^{1-\gamma} A_{i j} \in L^{\infty}(0, a) .
$$

Assume that there exists $\mathbf{W}=\left(W_{1}, W_{2}\right)^{T} \in\left(C^{2}(0, a] \cap C^{1}[0, a]\right)^{2}$, a solution of

$$
\left\{\begin{array}{l}
\mathbf{W}^{\prime \prime}(x)+A(x) \mathbf{W}(x)=\mathbf{0} \quad \text { in }(0, a], \\
\mathbf{W}(0)=\mathbf{W}^{\prime}(0)=\mathbf{0} .
\end{array}\right.
$$

Then $\mathbf{W} \equiv \mathbf{0}$ in $[0, a]$.

Proof. First we need the following result whose proof is a simple exercise of calculus.

Lemma 5.4. Let $f \in C(0, a] \cap L^{1+\delta}(0, a)$ for some $a, \delta>0$ and let $u \in C^{2}(0, a] \cap$ $C^{1}[0, a]$ be such that $u(0)=u^{\prime}(0)=0$ and $u^{\prime \prime}=f$ in $(0, a)$. Then

$$
u(x)=\int_{0}^{x}(x-t) f(t) d t, \quad \text { for all } 0 \leq x \leq a .
$$

Since $\mathbf{W} \in C^{1}[0,1] \times C^{1}[0,1]$ we have $A \mathbf{W} \in C(0, a] \cap L^{1+\delta}(0, a)$, provided that $0<\delta<-1-\gamma^{-1}$. Therefore, by Lemma 5.4 we get

$$
\mathbf{W}(x)=-\int_{0}^{x}(x-t) A(t) \mathbf{W}(t) d t \quad \text { for all } 0 \leq x \leq a .
$$

Define $B=\left(B_{i j}\right)_{1 \leq i, j \leq 2}$ by $B_{i j}(x)=x^{1-\gamma} A_{i j}(x), 0<x \leq a, 1 \leq i, j \leq 2$. Then $B_{i j} \in C(0, a] \cap L^{\infty}(0, a)$. Set

$$
M=\max _{1 \leq i, j \leq 2}\left\|B_{i j}\right\|_{\infty}, \quad k=\max \left\{\frac{|\mathbf{W}(x)|}{x} ; 0<x \leq a\right\},
$$


where $|\mathbf{W}(x)|=\max \left\{\left|W_{1}(x)\right|,\left|W_{2}(x)\right|\right\}$. Notice that both $M$ and $k$ are finite, since $\mathbf{W} \in C^{1}[0, a]$. From (5.7) we have

$$
\mathbf{W}(x)=-\int_{0}^{x}(x-t) B(t) \frac{\mathbf{W}(t)}{t} t^{\gamma} d t \quad \text { for all } 0 \leq x \leq a,
$$

which yields

$$
|\mathbf{W}(x)| \leq M \int_{0}^{x}(x-t) \frac{|\mathbf{W}(t)|}{t} t^{\gamma} d t \quad \text { for all } 0 \leq x \leq a .
$$

It follows that

$$
|\mathbf{W}(x)| \leq M k \int_{0}^{x}(x-t) t^{\gamma} d t=\frac{M k}{(1+\gamma)(2+\gamma)} x^{2+\gamma} \quad \text { for all } 0 \leq x \leq a .
$$

Using (5.9) in (5.8) we obtain

$$
\begin{aligned}
|\mathbf{W}(x)| & \leq \frac{M^{2} k}{(1+\gamma)(2+\gamma)} \int_{0}^{x}(x-t) t^{1+2 \gamma} d t \\
& =\frac{M^{2} k}{(1+\gamma)(2+\gamma)(2+2 \gamma)(3+2 \gamma)} x^{3+2 \gamma} \\
& \leq \frac{M^{2} k}{2(1+\gamma)^{2}} x^{3+2 \gamma} \quad \text { for all } 0 \leq x \leq a
\end{aligned}
$$

By induction, we deduce that for all $n \geq 2$ we have

$$
|\mathbf{W}(x)| \leq \frac{M^{n} k}{n !(1+\gamma)^{n}} x^{n+1+n \gamma} \quad \text { for all } 0 \leq x \leq a .
$$

Since $-1<\gamma \leq 0$, we can pass to the limit in the last inequality in order to get $\mathbf{W} \equiv \mathbf{0}$. This completes the proof.

\section{ACKNOWLEDGEMENT}

The author wishes to thank the referee for a careful reading of the manuscript and for helpful comments and suggestions.

\section{REFERENCES}

[1] Y.-S. Choi and J.P. McKenna, A singular Gierer-Meinhardt system of elliptic equations, Ann. Inst. H. Poincaré, Anal. Non Linéaire 17 (2000), no. 4, 503-522. MR1782742 (2001i:35072)

[2] _ A singular Gierer-Meinhardt system of elliptic equations: the classical case, Nonlinear Anal. 55 (2003), no. 5, 521-541. MR2012446 (2004k:35092)

[3] L. Dupaigne, M. Ghergu, and V. Rădulescu, Lane-Emden-Fowler equations with convection and singular potential, J. Math. Pures Appl. 87 (2007), 563-581. MR2335087

[4] M. Ghergu and V. Rădulescu, Singular elliptic problems: Bifurcation and asymptotic analysis, Oxford University Press, No. 37, 2008.

[5] _ A singular Gierer-Meinhardt system with different source terms, Proc. Roy. Soc. Edinburgh Sect. A, 138 (2008), no. 6, 1215-1234.

[6] _ On a class of singular Gierer-Meinhardt systems arising in morphogenesis, C. R. Math. Acad. Sci. Paris 344 (2007), no. 3, 163-168. MR2292281 (2007i:35054)

[7] - On a class of sublinear singular elliptic problems with convection term, J. Math. Anal. Appl. 311 (2005), no. 2, 635-646. MR2168423 (2006f:35086)

[8] A. Gierer and H. Meinhardt, A theory of biological pattern formation, Kybernetik 12 (1972), 30-39.

[9] C. Gui and F. Lin, Regularity of an elliptic problem with a singular nonlinearity, Proc. Roy. Soc. Edinburgh Sect. A 123 (1993), no. 6, 1021-1029. MR.1263903 (94m:35115)

[10] H. Jiang, Global existence of solutions of an activator-inhibitor system, Discrete Contin. Dyn. Syst. 14 (2006), 737-751. MR2177095 (2006g:35107) 
[11] J.P. Keener, Activators and inhibitors in pattern formation, Stud. Appl. Math. 59 (1978), 1-23. MR0479051 (57:18504)

[12] E.H. Kim, A class of singular Gierer-Meinhardt systems of elliptic boundary value problems, Nonlinear Anal. 59 (2004), 305-318. MR2093092 (2005g:35078)

[13] Singular Gierer-Meinhardt systems of elliptic boundary value problems, J. Math. Anal. Appl. 308 (2005), 1-10. MR2141599 (2006b:35081)

[14] D. Kinderlehrer and G. Stampacchia, An introduction to variational inequalities and their applications, Academic Press, New York, 1980. MR.567696 (81g:49013)

[15] A. Lazer and J.P. McKenna, On a singular nonlinear elliptic boundary value problem, Proc. Amer. Math. Soc. 111 (1991), no. 3, 721-730. MR.1037213 (91f:35099)

[16] W.-M. Ni, Diffusion, cross-diffusion, and their spike-layer steady states, Notices of Amer. Math. Soc. 45 (1998), no. 3-4, 9-18. MR.1490535 (99a:35132)

[17] _ Diffusion and cross-diffusion in pattern formation, Atti Accad. Naz. Lincei Cl. Sci. Fis. Mat. Natur. Rend. Lincei Mat. Appl. 15 (2004), no. 3-4, 197-214. MR2148879 (2006c:35092)

[18] W.-M. Ni, K. Suzuki, and I. Takagi, The dynamics of a kynetics activator-inhibitor system, J. Differential Equations 229 (2006), no. 2, 426-465. MR2263562 (2007h:35154)

[19] W.-M. Ni and I. Takagi, On the shape of least-energy solutions to a semilinear Neumann problem, Comm. Pure Appl. Math. 44 (1991), 819-851. MR.1115095 (92i:35052)

[20] L L L Lating the peaks of least-energy solutions to a semilinear Neumann problem, Duke Math. J. 70 (1993), 247-281. MR.1219814 (94h:35072)

[21] W.-M. Ni and J. Wei, On positive solutions concentrating on spheres for the Gierer-Meinhardt system, J. Differential Equations 221 (2006), no. 1, 158-189. MR2193846 (2007a:35027)

[22] S.D. Taliaferro, A nonlinear singular boundary value problem, Nonlinear Anal., T.M.A. 3 (1979), no. 6, 897-904. MR0548961 (81i:34011)

[23] A.M. Turing, The chemical basis of morphogenesis, Philosophical Transactions of the Royal Society (B) 237 (1952), 37-72.

[24] A. Trembley, Mémoires pour servir à l'histoire d'un genre de polype d'eau douce, à bras en forme de corne, Verbeek, Leiden, Netherland, 1744.

[25] J. Wei and M. Winter, Spikes for the Gierer-Meinhardt system in two dimensions: the strong coupling case, J. Differential Equations 178 (2002), no. 2, 478-518. MR.1879835 (2002m:35095)

[26] _ Existence and stability analysis of asymmetric patterns for the Gierer-Meinhardt system, J. Math. Pures Appl. 83 (2004), no. 4, 433-476. MR2048385 (2005i:35074)

[27] Z. Wei, Positive solutions of singular sublinear second order boundary value problems, Systems Sci. Math. Sci. 11 (1998), no. 1, 82-88. MR1610532 (98k:34033)

[28] Z. Zhang and J. Cheng, Existence and optimal estimates of solutions for singular nonlinear Dirichlet problems, Nonlinear Anal. 57 (2004), no. 3, 473-484. MR2064102(2005c:35114)

Institute of Mathematics "Simion Stollow" of the Romanian Academy, P.O. Box 1764, RO-014700 Bucharest, Romania

E-mail address: marius.ghergu@imar.ro 